\title{
Molecular gas and dust properties of galaxies from the Great Observatories All-sky LIRG Survey ${ }^{\star}$
}

R. Herrero-Illana ${ }^{1,2}$, G. C. Privon ${ }^{3,4}$, A. S. Evans ${ }^{5,6}$, T. Díaz-Santos ${ }^{7}$, M. Á. Pérez-Torres ${ }^{2,8}$, V. U ${ }^{9}$, A. Alberdi ${ }^{2}$, K. Iwasawa ${ }^{10}$, L. Armus ${ }^{11}$, S. Aalto ${ }^{12}$, J. Mazzarella ${ }^{13}$, J. Chu ${ }^{14}$, D. B. Sanders ${ }^{15,16}$, L. Barcos-Muñoz ${ }^{5,6}$, V. Charmandaris ${ }^{17}$, S. T. Linden ${ }^{5}$, I. Yoon ${ }^{6}$, D. T. Frayer ${ }^{18}$, H. Inami ${ }^{19}$, D.-C. Kim ${ }^{6}$, H. J. Borish ${ }^{5}$, J. Conway ${ }^{12}$, E. J. Murphy ${ }^{6,13}$, Y. Song ${ }^{5}$, S. Stierwalt ${ }^{11}$, and J. Surace ${ }^{11}$

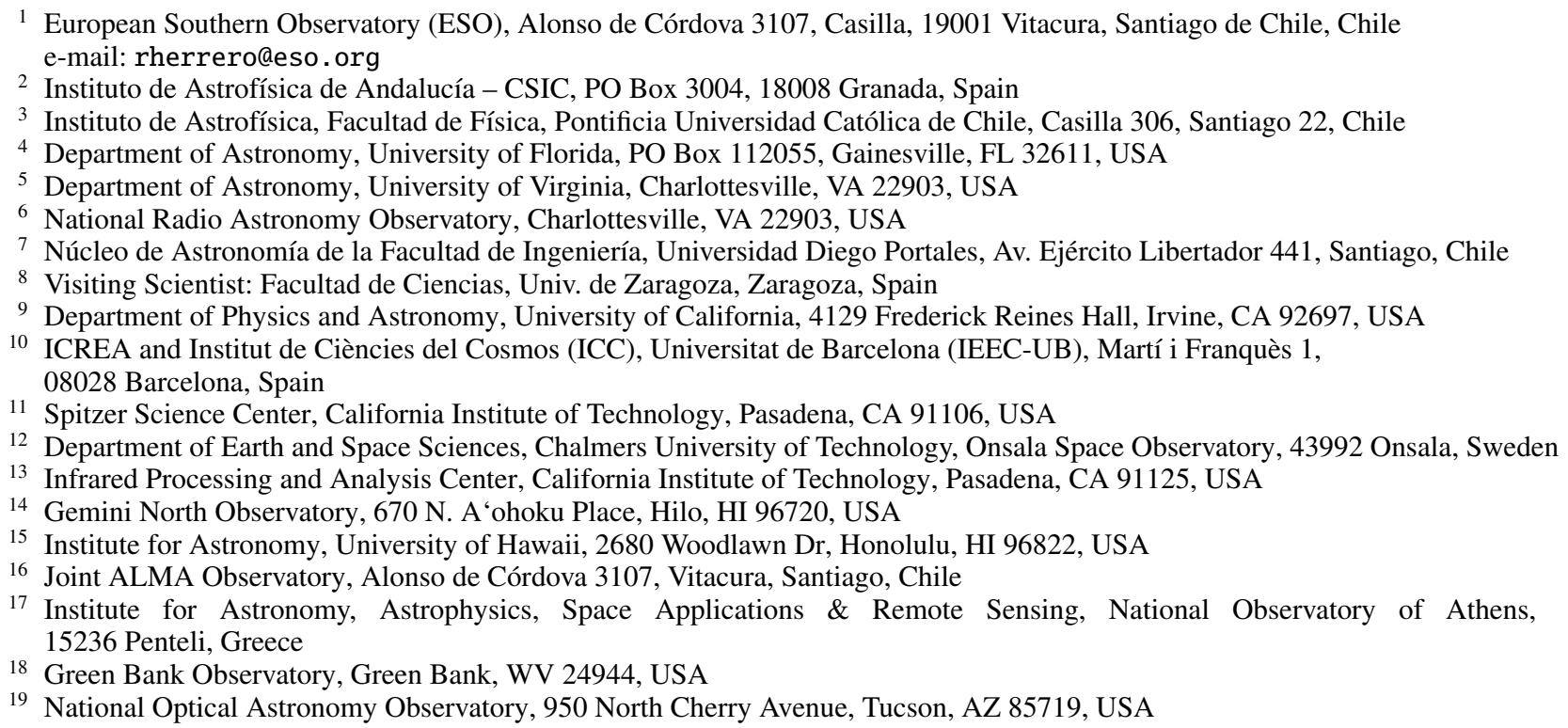

Received 14 August 2018 / Accepted 29 June 2019

\section{ABSTRACT}

We present IRAM-30 m Telescope ${ }^{12} \mathrm{CO}$ and ${ }^{13} \mathrm{CO}$ observations of a sample of 55 luminous and ultraluminous infrared galaxies (LIRGs and ULIRGs) in the local universe. This sample is a subset of the Great Observatory All-Sky LIRG Survey (GOALS), for which we use ancillary multi-wavelength data to better understand their interstellar medium and star formation properties. Fifty-three $(96 \%)$ of the galaxies are detected in ${ }^{12} \mathrm{CO}$, and $29(52 \%)$ are also detected in ${ }^{13} \mathrm{CO}$ above a $3 \sigma$ level. The median full width at zero intensity (FWZI) velocity of the CO line emission is $661 \mathrm{~km} \mathrm{~s}^{-1}$, and $\sim 54 \%$ of the galaxies show a multi-peak CO profile. Herschel photometric data is used to construct the far-IR spectral energy distribution of each galaxy, which are fit with a modified blackbody model that allows us to derive dust temperatures and masses, and infrared luminosities. We make the assumption that the gas-to-dust mass ratio of (U)LIRGs is comparable to local spiral galaxies with a similar stellar mass (i.e., gas/dust of mergers is comparable to their progenitors) to derive a CO-to- $\mathrm{H}_{2}$ conversion factor of $\langle\alpha\rangle=1.8_{-0.8}^{+1.3} M_{\odot}\left(\mathrm{K} \mathrm{km} \mathrm{s}^{-1} \mathrm{pc}^{2}\right)^{-1}$; such a value is comparable to that derived for (U)LIRGs based on dynamical mass arguments. We derive gas depletion times of 400-600 Myr for the (U)LIRGs, compared to the $1.3 \mathrm{Gyr}$ for local spiral galaxies. Finally, we re-examine the relationship between the ${ }^{12} \mathrm{CO} /{ }^{13} \mathrm{CO}$ ratio and dust temperature, confirming a transition to elevated ratios in warmer systems.

Key words. ISM: molecules - galaxies: ISM - galaxies: active - galaxies: starburst - radio lines: galaxies

\section{Introduction}

Luminous and ultraluminous infrared galaxies (LIRGs: $L_{\mathrm{IR}}(8-$ $1000 \mu \mathrm{m})>10^{11} L_{\odot} ;$ ULIRGs; $\left.L_{\mathrm{IR}}>10^{12} L_{\odot}\right)$ are known to host powerful starbursts, making them ideal for studying the

* A copy of the reduced spectra is available at the CDS via anonymous ftp to cdsarc.u-strasbg.fr (130.79.128.5) or via http: //cdsarc.u-strasbg.fr/viz-bin/qcat?]/A+A/628/A71 properties of extreme star-forming environments. Since the discovery of (U)LIRGs as an important galaxy population by the Infrared Astronomical Satellite (IRAS), which found that $30 \%-50 \%$ of the total bolometric luminosity of galaxies in the local universe is emitted at infrared (IR) and submillimeter wavelengths (Soifer et al. 1986), CO observations of (U)LIRGs have been carried out to characterize the molecular gas responsible for the ongoing star formation, as well as to trace galaxy kinematics (e.g., Tinney et al. 1990; Sanders et al. 1991; 
Aalto et al. 1995; Solomon et al. 1997; Downes \& Solomon 1998; Bryant \& Scoville 1999; Yao et al. 2003; Narayanan et al. 2005; Papadopoulos et al. 2012a; Yamashita et al. 2017). Much of the interpretation of the data is reliant on the CO luminosityto-molecular gas mass conversion factor, $\alpha=M\left(\mathrm{H}_{2}\right) / L_{\mathrm{CO}}^{\prime}$; the commonly used "Milky Way" conversion factor, $\alpha_{\mathrm{MW}} \simeq$ $4 M_{\odot}\left(\mathrm{K} \mathrm{km} \mathrm{s}^{-1} \mathrm{pc}^{2}\right)^{-1}$, has been called into question for its use with (U)LIRGs, for which alternative values have been proposed (e.g., Downes \& Solomon 1998; Bolatto et al. 2013). The choice directly impacts upon estimates of the total gas mass, the star formation efficiency, and the gas depletion timescale, which can differ by almost an order of magnitude. In addition to $\mathrm{CO}$, observations of high density and optically thin gas tracers have provided the opportunity to better assess the physical conditions of the interstellar medium (ISM), in part by determining the physical state of the gas more directly involved in the formation of future stars.

The advent of broadband receivers on millimeter-wave telescopes has enabled the detection of multiple lines at once, probing a range of optical depths, critical densities, and temperatures. In our present study, we make use of the $30 \mathrm{~m}$ Telescope of the Institut de Radioastronomie Millimétrique (IRAM-30 m) to detect multiple millimeter-wave lines from a sample of 55 nearby (U)LIRGs selected from the Great Observatories All-Sky LIRG Survey (GOALS, Armus et al. 2009). We focus here primarily on the ${ }^{12} \mathrm{CO}(1 \rightarrow 0)$ and ${ }^{13} \mathrm{CO}(1 \rightarrow 0)$ data, which is combined with new Herschel Space Observatory observations in order to estimate the conversion factor $\alpha$ from an assumed gas-to-dust mass ratio, the star formation efficiency, and gas depletion timescales, and to examine the relation between the ${ }^{12} \mathrm{CO}(1 \rightarrow 0)$-to- ${ }^{13} \mathrm{CO}(1 \rightarrow 0)$ ratio and the dust temperature. A complementary study of dense gas tracers $\mathrm{HCN}(1 \rightarrow 0)$ and $\mathrm{HCO}^{+}(1 \rightarrow 0)$, observed as part of this IRAM campaign, was presented in Privon et al. (2015).

This paper is organized as follows: we describe our sample in Sect. 2. Observations and data reduction are summarized in Sect. 3. The main results are presented in Sect. 4. In Sect. 5 we determine the CO-to- $\mathrm{H}_{2}$ conversion factor Sect. 5.1, derive the star formation properties of the sample Sect. 5.3, and reexamine the ${ }^{12} \mathrm{CO} /{ }^{13} \mathrm{CO}$ relationship with dust temperature Sect. 5.4. Finally, we summarize our results in Sect. 6. Throughout this study, we adopt a Wilkinson Microwave Anisotropy Probe (WMAP) cosmology of $H_{0}=69.3 \mathrm{~km} \mathrm{~s}^{-1} \mathrm{Mpc}^{-1}, \Omega_{\Lambda}=$ 0.714 and $\Omega_{\mathrm{m}}=0.286$ (Hinshaw et al. 2013).

\section{Sample}

Our sources are selected from the GOALS sample, which consists of all 180 luminous and 22 ultraluminous nearby IR galaxy systems in the IRAS Revised Bright Galaxy Sample $\left(f_{60 \mu \mathrm{m}}>5.24 \mathrm{Jy}\right.$ : Sanders et al. 2003). The GOALS sample has been observed with the Hubble Space Telescope (HST; Haan et al. 2011; Kim et al. 2013), the Spitzer Space Telescope (Díaz-Santos et al. 2010, 2011; Petric et al. 2011; Stierwalt et al. 2013, 2014), the Herschel Space Observatory (Chu et al. 2017), the Galaxy Evolution Explorer (GALEX; Howell et al. 2010), and the Chandra X-ray Observatory (Iwasawa et al. 2011), making it the most complete multiwavelength sample of nearby, IR-bright galaxies. In addition, ground-based $\mathrm{HI}$, radio, and near-IR spectroscopic data complement the space-telescopebased dataset. GOALS contains a sufficient number of LIRGs to cover the full range of galaxy-galaxy interactions and merger stages, including isolated sources. The sample of 55 (U)LIRGs observed for our present CO survey consists of GOALS sources accessible from Pico Veleta that had not been previously observed by the IRAM- $30 \mathrm{~m}$ Telescope. Unfortunately, due to the lack of a public archive for the IRAM-30 m Telescope, previously observed data for GOALS galaxies cannot be obtained in its raw format, so a systematic data reduction could not be guaranteed. For this reason, we restricted the current study to the aforementioned sample of 55 (U)LIRGs.

In the observed sample, 48 of the galaxies are LIRGs or components of LIRGs, and seven are ULIRGs or component of ULIRGs. In the cases where each component of a system was observed independently, these were treated as individual sources throughout the analysis. The list of galaxies is presented in Table 1, along with $L_{\mathrm{IR}}$ from Armus et al. (2009). As shown in the histogram of Fig. 1, our observed sources are representative of the complete GOALS sample in terms of luminosity.

To compare our (U)LIRG sample with local spiral galaxies, we have used published observations from the HERA CO-Line Extragalactic Survey (HERACLES; Leroy et al. 2009) and the Five College Radio Astronomy Observatory (FCRAO; Young et al. 1995), for which CO and Herschel data are available. These include a total of 22 sources.

For comparison, our (U)LIRG sample has a median redshift (see Sect. 4 for details) of $0.0248 \pm 0.0163$ and a median $\log \left(L_{\mathrm{IR}} / L_{\odot}\right)$ of $(11.45 \pm 0.33)$ according to Armus et al. (2009), where the errors indicate the standard deviation of the sample. On the other hand, the local comparison sample (both HERACLES and FCRAO) has a median redshift of $0.0023 \pm 0.0018$ and a median $\log \left(L_{\mathrm{IR}} / L_{\odot}\right)$ of $10.28 \pm 0.51$.

\section{Observations and data reduction}

The observations ${ }^{1}$ presented in this study were carried out with the Eight Mixer Receiver (EMIR) multiband millimeterwave receiver (Carter et al. 2012) at the IRAM-30 m Telescope on Pico Veleta, Spain, in five observing periods: June 2010, September 2011, December 2011, October 2012 (PI: K. Iwasawa), and March 2014 (PI: R. Herrero-Illana). The final March 2014 run was obtained during the director's discretionary time in order to verify the ${ }^{13} \mathrm{CO}$ line strength of three sources (IRAS F05189-2524, IRAS 22491-1808, and IRAS 07251-0248). We tuned the receiver to two frequency windows: one centered at the redshifted frequencies of $\mathrm{HCN}(1 \rightarrow 0)$ and $\mathrm{HCO}^{+}(1 \rightarrow 0)$ (rest frequencies of 88.632 and $89.189 \mathrm{GHz}$, respectively; presented in Privon et al. 2015), and the other at the frequencies of ${ }^{12} \mathrm{CO}(1 \rightarrow 0)$ and ${ }^{13} \mathrm{CO}(1 \rightarrow 0)$ (rest frequencies of 115.271 and $110.201 \mathrm{GHz}$, respectively). The EMIR receiver has a total bandwidth of $8 \mathrm{GHz}$. All the observations were performed in wobbler switching mode. For the majority of the galaxies, the peak of their Spitzer IRAC channel 4 (i.e., $8 \mu \mathrm{m}$ ) emission was used as the pointing center. A complete journal of observations is presented in Table 2, along with the measured line intensities.

The data were reduced using the Continuum and Line Analysis Single-dish Software $\left(\right.$ CLASS) ${ }^{2}$ package. For each scan, gain elevation calibration, platforming correction (when the Fast Fourier Transform Spectrometer (FTS) backend was used), and order one baseline subtraction were performed. Then, the scans for each galaxy were averaged together and the average spectrum was smoothed to $\sim 25 \mathrm{~km} \mathrm{~s}^{-1}$ resolution. A final linear baseline was subtracted over the channels that did not contain

\footnotetext{
1 Based on observations carried out with the IRAM-30 m Telescope. IRAM is supported by INSU/CNRS (France), MPG (Germany) and IGN (Spain).

2 http://iram.fr/IRAMFR/GILDAS/
} 
Table 1. Source list.

\begin{tabular}{|c|c|c|c|}
\hline Source name & $\begin{array}{c}\mathrm{RA} \\
(\mathrm{J} 2000) \\
\end{array}$ & $\begin{array}{c}\text { Dec } \\
(\mathrm{J} 2000)\end{array}$ & $\begin{array}{c}\log \left(L_{\mathrm{IR}}\right) \\
\left(L_{\odot}\right)\end{array}$ \\
\hline NGC 0034 & 001106.55 & -120627.90 & 11.49 \\
\hline Arp $256 \mathrm{~N}$ & 001850.17 & -102144.78 & $11.48^{(\dagger)}$ \\
\hline Arp 256S & 001850.90 & -102236.19 & $11.48^{(\dagger)}$ \\
\hline IC 1623 & 010747.53 & -173025.88 & 11.71 \\
\hline MCG -03-04-014 & 011008.96 & -165109.79 & 11.65 \\
\hline IRAS F01364-1042 & 013852.92 & -102711.41 & 11.85 \\
\hline IC 0214 & 021405.47 & 051025.21 & 11.43 \\
\hline UGC 01845 & 022407.99 & 475810.81 & 11.12 \\
\hline NGC 0958 & 023042.85 & -025620.51 & 11.20 \\
\hline ESO 550-IG025 & 042120.00 & -184839.38 & 11.51 \\
\hline UGC 03094 & 043533.83 & 191018.19 & 11.41 \\
\hline NGC 1797 & 050744.85 & -080108.69 & 11.04 \\
\hline IRAS F05189-2524 & 052101.47 & -252145.40 & 12.16 \\
\hline IRAS F05187-1017 & 052106.54 & -101446.79 & 11.30 \\
\hline IRAS F06076-2139 & 060945.81 & -214023.70 & 11.65 \\
\hline NGC 2341 & 070912.00 & 203610.01 & 11.17 \\
\hline NGC 2342 & 070918.06 & 203810.39 & 11.31 \\
\hline IRAS 07251-0248 & 072737.55 & -025454.11 & 12.39 \\
\hline IRAS F09111-1007 W & 091336.40 & -101930.00 & $12.06^{(\dagger)}$ \\
\hline IRAS F09111-1007 E & 091338.80 & -101920.32 & $12.06^{(\dagger)}$ \\
\hline UGC 05101 & 093551.60 & 612111.81 & 12.01 \\
\hline 2MASX J11210825-0259399 (1) & 112108.28 & -025939.01 & $11.43^{(\dagger)}$ \\
\hline CGCG 011-076 & 112112.22 & -025902.18 & $11.43^{(\dagger)}$ \\
\hline IRAS F12224-0624 & 122503.89 & -064051.71 & 11.36 \\
\hline CGCG 043-099 & 130150.80 & 041959.99 & 11.68 \\
\hline ESO 507-G070 & 130252.35 & -235517.69 & 11.56 \\
\hline NGC 5104 & 132123.10 & 002032.89 & 11.27 \\
\hline IC 4280 & 133253.30 & -241225.88 & 11.15 \\
\hline NGC 5258 & 133957.25 & 004947.60 & $11.62^{(\dagger)}$ \\
\hline UGC 08739 & 134913.91 & 351526.21 & 11.15 \\
\hline NGC 5331 & 135216.20 & 020605.62 & 11.66 \\
\hline CGCG 247-020 & 141943.21 & 491411.90 & 11.39 \\
\hline IRAS F14348-1447 & 143738.34 & -150022.79 & 12.39 \\
\hline CGCG 049-057 & 151313.09 & 071332.02 & 11.35 \\
\hline NGC 5936 & 153000.86 & 125922.20 & 11.14 \\
\hline IRAS F16164-0746 & 161911.79 & -075402.81 & 11.62 \\
\hline CGCG 052-037 & 163056.50 & 040458.51 & 11.45 \\
\hline IRAS F16399-0937 & 164240.21 & -094314.41 & 11.63 \\
\hline NGC 6285 & 165823.99 & 585721.31 & $11.37^{(\dagger)}$ \\
\hline NGC 6286 & 165831.55 & 585612.19 & $11.37^{(\dagger)}$ \\
\hline IRAS F17138-1017 & 171635.76 & -102039.80 & 11.49 \\
\hline UGC 11041 & 175451.83 & 344634.50 & 11.11 \\
\hline CGCG 141-034 & 175656.63 & 240101.31 & 11.20 \\
\hline IRAS $18090+0130$ & 181138.41 & 013140.12 & 11.65 \\
\hline NGC 6701 & 184312.50 & 603911.20 & 11.12 \\
\hline NGC 6786 & 191054.00 & 732435.71 & $11.49^{(\dagger)}$ \\
\hline UGC 11415 & 191104.40 & 732532.02 & $11.49^{(\dagger)}$ \\
\hline ESO 593-IG008 & 191431.15 & -211906.31 & 11.93 \\
\hline NGC 6907 & 202506.60 & -244832.11 & 11.11 \\
\hline IRAS $21101+5810$ & 211130.40 & 582303.19 & 11.81 \\
\hline ESO 602-G025 & 223125.49 & -190204.31 & 11.34 \\
\hline UGC 12150 & 224112.18 & 341457.01 & 11.35 \\
\hline IRAS F22491-1808 & 225149.36 & -175224.82 & 12.20 \\
\hline CGCG $453-062$ & 230456.55 & 193307.09 & 11.38 \\
\hline 2MASX J23181352+0633267 (2) & 231813.52 & 063326.50 & $11.12^{(\dagger)}$ \\
\hline
\end{tabular}

Notes. Coordinates refer to the pointing position of our observations. ${ }^{(1)}$ Southwest component of CGCG 011-076. ${ }^{(2)}$ Southwest component of NGC 7591. ${ }^{(\dagger)}$ Component of a system. In these cases, the shown $L_{\mathrm{IR}}$ refers to the whole system.

line emission. We obtained line fluxes by integrating all channels within the line profile. To account for systematic uncertainties, we added a standard $10 \%$ of error in quadrature to the rms of the fluxes, which is the uncertainty for IRAM-30 m data as estimated by the observatory ${ }^{3}$. Figure 2 contains an example of a

\footnotetext{
3 http://www.iram.fr/GENERAL/calls/s17/ 30mCapabilities.pdf
}

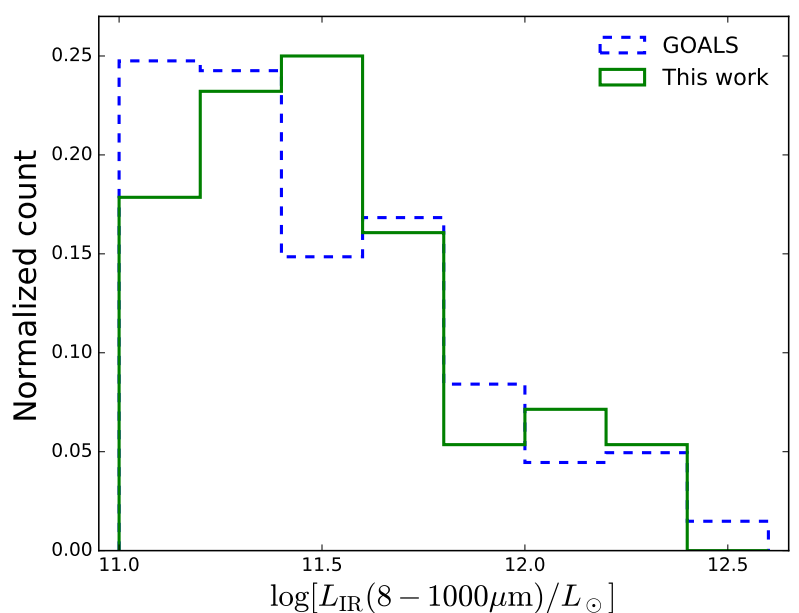

Fig. 1. Normalized histogram comparing $L_{\mathrm{IR}}$ from the complete set of 202 GOALS galaxies and our observed sample. All $L_{\mathrm{IR}}$ were obtained from Armus et al. (2009).

resultant spectrum, and the spectra of the remaining galaxies are shown in Appendix A.

\section{Results}

In Tables 2 and 3 we present the measured and derived properties from the IRAM observations, respectively. From the 55 observed sources we detect ${ }^{12} \mathrm{CO}$ in $53(\sim 96 \%)$ and ${ }^{13} \mathrm{CO}$ in $29(\sim 52 \%)$, above a $3 \sigma$ level. The only two sources with no ${ }^{12} \mathrm{CO}$ detection (2MASX J11210825-0259399 and 2MASX J23181352+0633267) are subcomponents of LIRGs and, given the non-detections, were excluded from the analysis. Line intensities and luminosities for ${ }^{13} \mathrm{CO}$ were obtained by integrating over the velocity range corresponding to the ${ }^{12} \mathrm{CO}$ detections. We find that $\sim 54 \%$ of the sources show a multiple peak profile (see Figs. 2 and A.1). For that reason, the full width at half maximum (FWHM) cannot be clearly determined, so we calculate the full width at zero intensity (FWZI), which was obtained as the width where the sum of the fitted Gaussians of each source (see Fig. 2) is above $0.5 \mathrm{mK}$. The FWZI of our sample has a median value of $661 \mathrm{~km} \mathrm{~s}^{-1}$.

We derived the integrated $\mathrm{CO}$ luminosities, measured in $\mathrm{K} \mathrm{km} \mathrm{s}^{-1} \mathrm{pc}^{2}$, via

$L_{\mathrm{CO}}^{\prime}=3.25 \times 10^{7}\left(S_{\mathrm{CO}} \Delta v\right) v_{\mathrm{obs}}^{-2} D_{L}^{2}(1+z)^{-3}$

(Solomon et al. 1992), where $S_{\mathrm{CO}} \Delta v$ is the velocity integrated flux $\left(\mathrm{Jy} \mathrm{km} \mathrm{s}^{-1}\right), v_{\mathrm{obs}}$ is the observed frequency $(\mathrm{GHz}), D_{\mathrm{L}}$ is the luminosity distance (Mpc), and $z$ is the redshift of each source, which was obtained from a visual determination of the center of the spectral profile. We use a point source sensitivity of $S / T_{A}^{*}=$ $6.1 \mathrm{Jy} \mathrm{K}^{-1}$. Considering that $v_{\text {rest }}=v_{\text {obs }}(1+z)$ and substituting $v_{\text {rest }}\left({ }^{12} \mathrm{CO}\right)=115.271 \mathrm{GHz}$ and $v_{\text {rest }}\left({ }^{13} \mathrm{CO}\right)=110.201 \mathrm{GHz}$, we can rewrite Eq. (1) in the useful form

$L_{n}^{\prime} \mathrm{CO}=A_{n \mathrm{CO}} \times 10^{3}\left(\frac{S_{\mathrm{CO}} \Delta v}{\mathrm{Jy} \mathrm{km} \mathrm{s}^{-1}}\right)\left(\frac{D_{\mathrm{L}}}{\mathrm{Mpc}}\right)^{2}(1+z)^{-1}$,

where $A_{12} \mathrm{CO}=2.45$ and $A_{{ }^{13} \mathrm{CO}}=2.68$.

Three sources in our sample have available archive CO observations (Sanders et al. 1991) with the National Radio Astronomy Observatory (NRAO) $12 \mathrm{~m}$ telescope. Their CO flux for IRAS 22491-1808 $\left((55.0 \pm 11.0) \times 10^{8} \mathrm{~K} \mathrm{~km} \mathrm{~s}^{-1} \mathrm{pc}^{2}\right)$ 

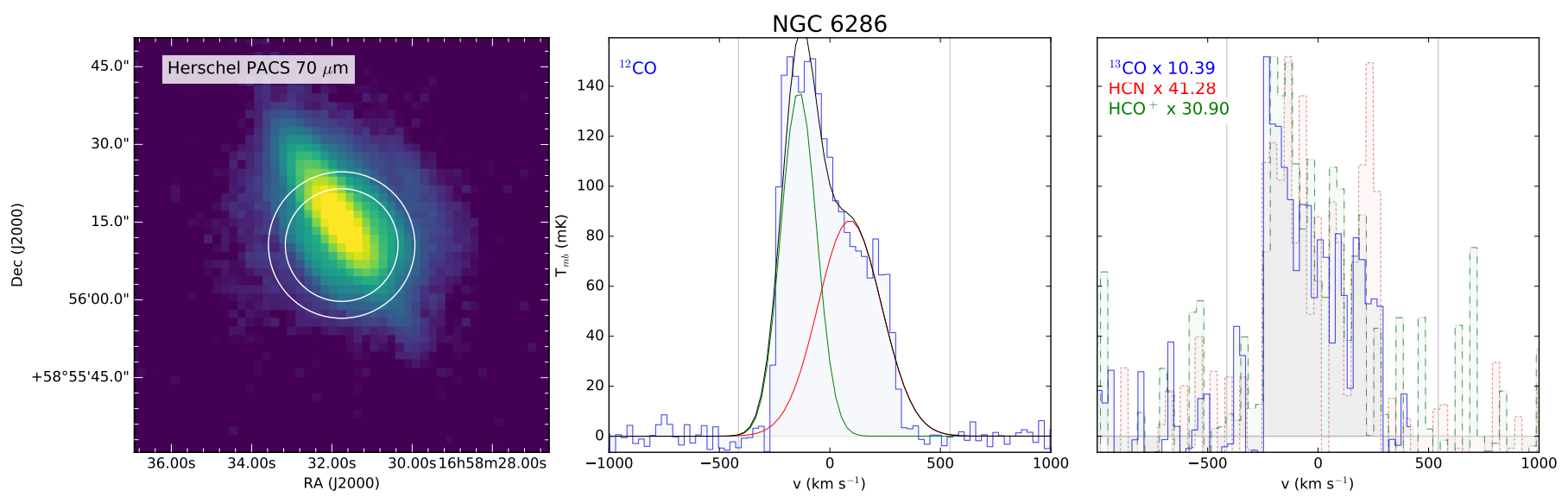

Fig. 2. Spectral line profiles for NGC 6286. Left panel: Herschel PACS $70 \mu \mathrm{m}$ map (see Chu et al. 2017) with a logarithmic stretch to highlight the full extent of the infrared emission relative to the IRAM-30 m Telescope. Overlaid circles represent the pointing position and beam sizes (FWHM) corresponding to the ${ }^{12} \mathrm{CO}$ and ${ }^{13} \mathrm{CO}$ (inner), and $\mathrm{HCN}$ and $\mathrm{HCO}^{+}$(outer) IRAM observations. The middle panel shows the continuum-subtracted $\mathrm{CO}$ spectra, fitted with Gaussian components. Velocity frame is defined according to the redshifts quoted in Table 3. Gray vertical lines correspond to the velocity integration limits of the FWZI. Right panel: continuum-subtracted spectra of ${ }^{13} \mathrm{CO}$ (blue, solid line), $\mathrm{HCN}$ (red, dotted line), and $\mathrm{HCO}^{+}$(green, dashed line) in the same velocity range as the $\mathrm{CO}$ spectrum. The spectra have been normalized to the peak of the CO line, and the normalization factor is shown for each line. The complete data set with all the observations, as well as a table with the fitted Gaussian components of the ${ }^{12} \mathrm{CO}$ spectra is shown in Appendix A.

Table 2. Measured parameters.

\begin{tabular}{|c|c|c|c|c|c|c|}
\hline Source name & $\begin{array}{l}\text { Date } \\
(2)\end{array}$ & $\begin{array}{c}t_{\text {int }} \\
(\min ) \\
(3)\end{array}$ & $\begin{array}{l}T_{\text {sys }} \\
(\mathrm{K}) \\
(4) \\
\end{array}$ & $\begin{array}{c}S_{{ }^{12} \mathrm{CO} \Delta v} \\
\left(\mathrm{Jy} \mathrm{km} \mathrm{s}^{-1}\right) \\
(5)\end{array}$ & $\begin{array}{c}S_{{ }_{13} \mathrm{CO}} \Delta v \\
\left(\mathrm{Jy} \mathrm{km} \mathrm{s}^{-1}\right) \\
(6)\end{array}$ & $\begin{array}{c}\text { FWZI } \\
\left(\mathrm{km} \mathrm{s}^{-1}\right) \\
(7)\end{array}$ \\
\hline NGC 0034 & 2011-09 & 10.5 & 278,182 & $126.0 \pm 3.6$ & $<5.4$ & 763.5 \\
\hline Arp $256 \mathrm{~N}$ & $2011-12$ & 21.0 & 188,138 & $15.1 \pm 1.3$ & $4.6 \pm 1.3$ & 313.1 \\
\hline Arp $256 \mathrm{~S}$ & 2011-12 & 16.0 & 197,142 & $45.8 \pm 1.6$ & $<4.8$ & 495.7 \\
\hline IC 1623 & 2011-12 & 21.5 & 200,143 & $469.2 \pm 1.9$ & $10.9 \pm 1.3$ & 699.5 \\
\hline MCG -03-04-014 & 2011-12 & 16.0 & 175,133 & $96.0 \pm 1.2$ & $<5.4$ & 578.3 \\
\hline IRAS F01364-1042 & 2011-09 & 21.0 & 171,135 & $37.4 \pm 1.4$ & $<4.2$ & 763.3 \\
\hline IC 0214 & 2011-12 & 16.0 & 183,177 & $62.8 \pm 1.7$ & $5.9 \pm 1.1$ & 549.5 \\
\hline UGC 01845 & $2012-10$ & 10.0 & 204,140 & $208.0 \pm 2.5$ & $11.3 \pm 1.9$ & 670.7 \\
\hline NGC 0958 & $2012-10$ & 15.5 & 285,176 & $144.1 \pm 4.2$ & $12.1 \pm 2.1$ & 854.3 \\
\hline ESO 550-IG025 & 2011-09 & 16.0 & 222,157 & $70.3 \pm 2.7$ & $<4.2$ & 751.6 \\
\hline UGC 03094 & $2012-10$ & 10.0 & 197,136 & $130.9 \pm 2.8$ & $12.7 \pm 1.4$ & 858.6 \\
\hline NGC 1797 & $2012-10$ & 15.5 & 219,148 & $126.4 \pm 2.2$ & $7.3 \pm 1.1$ & 541.4 \\
\hline IRAS F05189-2524 & 2014-03 & 128.0 & 236,173 & $31.0 \pm 0.5$ & $<1.5$ & 423.8 \\
\hline IRAS F05187-1017 & 2014-03 & 51.0 & 166,117 & $62.0 \pm 0.6$ & $3.2 \pm 0.6$ & 600.9 \\
\hline IRAS F06076-2139 & 2011-09 & 16.0 & 200,145 & $37.6 \pm 1.7$ & $<3.3$ & 539.7 \\
\hline NGC 2341 & $2014-03$ & 25.5 & 235,150 & $105.0 \pm 2.3$ & $6.8 \pm 1.2$ & 568.3 \\
\hline NGC 2342 & $2012-10$ & 15.5 & 169,117 & $111.5 \pm 1.7$ & $7.2 \pm 1.2$ & 568.6 \\
\hline IRAS $07251-0248$ & 2014-03 & 21.5 & 136,106 & $18.0 \pm 1.4$ & $<2.1$ & 763.8 \\
\hline IRAS F09111-1007 W & 2011-09 & 21.5 & $184, \ldots(\dagger)$ & $38.5 \pm 1.1$ & $\ldots$ & 493.5 \\
\hline IRAS F09111-1007 E & 2011-09 & 21.5 & $189, \ldots(\dagger)$ & $19.2 \pm 1.9$ & & 630.9 \\
\hline UGC 05101 & 2010-06 & 53.0 & 159,122 & $86.6 \pm 1.5$ & $<2.4$ & 973.1 \\
\hline 2MASX J11210825-0259399 & 2014-03 & 30.5 & 203,134 & 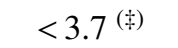 & $<3.7(\doteqdot)$ & $\ldots$ \\
\hline CGCG 011-076 & $2012-10$ & 10.0 & 201,179 & $114.3 \pm 2.6$ & $8.3 \pm 2.6$ & 728.8 \\
\hline IRAS F12224-0624 & $2014-03$ & 20.5 & 190,126 & $19.9 \pm 0.9$ & $<2.7$ & 312.8 \\
\hline CGCG 043-099 & 2011-09 & 26.5 & 194,141 & $72.8 \pm 1.3$ & $4.9 \pm 1.3$ & 701.5 \\
\hline ESO 507-G070 & 2011-12 & 10.5 & 227,135 & $126.2 \pm 3.6$ & $<4.5$ & 882.0 \\
\hline NGC 5104 & $2012-10$ & 10.0 & 186,151 & $131.6 \pm 2.9$ & $9.1 \pm 2.2$ & 879.6 \\
\hline IC 4280 & $2012-10$ & 10.0 & 252,178 & $119.7 \pm 3.5$ & $8.4 \pm 1.8$ & 593.6 \\
\hline NGC 5258 & 2011-12 & 10.5 & 243,145 & $128.6 \pm 3.2$ & $9.9 \pm 1.9$ & 675.5 \\
\hline
\end{tabular}

Notes. (1) Source name; (2) Observing date; (3) Integration time; (4) System temperatures for ${ }^{12} \mathrm{CO}$ and ${ }^{13} \mathrm{CO}$, respectively; (5) Line intensity for ${ }^{12} \mathrm{CO}$; (6) Line intensity for ${ }^{13} \mathrm{CO}$. In those cases where the detection was not significant, the $3 \sigma$ upper limit is quoted. (7) Full width at zero intensity. ${ }^{(\dagger) 13} \mathrm{CO}$ line was not observed for these sources due to a non-optimal receiver setup. ${ }^{(\ddagger)}$ To obtain the upper limits for the ${ }^{12} \mathrm{CO}$ and ${ }^{13} \mathrm{CO}$ non-detections, we have assumed a FWZI corresponding to the median of the sample. 
Table 2. continued.

\begin{tabular}{lcccccc}
\hline \hline Source name & Date & $\begin{array}{c}t_{\text {int }} \\
(\mathrm{min})\end{array}$ & $\begin{array}{c}T_{\text {sys }} \\
(\mathrm{K})\end{array}$ & $\begin{array}{c}S_{12} \mathrm{CO} \Delta v \\
\left(\mathrm{Jy} \mathrm{km} \mathrm{s}^{-1}\right)\end{array}$ & $\begin{array}{c}S_{13} \mathrm{CO} \Delta v \\
\left(\mathrm{Jy} \mathrm{km} \mathrm{s}^{-1}\right) \\
(1)\end{array}$ & $\begin{array}{c}\text { FWZI } \\
\left(\mathrm{km} \mathrm{s}^{-1}\right)\end{array}$ \\
\hline UGC 08739 & $(3)$ & $(4)$ & $(5)$ & $(6)$ & $(7)$ \\
NGC 5331 & $2012-10$ & 15.5 & 232,166 & $208.4 \pm 2.7$ & $14.2 \pm 1.3$ & 774.8 \\
CGCG 247-020 & $2011-09$ & 16.0 & 176,134 & $119.7 \pm 2.5$ & $8.6 \pm 1.7$ & 1155.3 \\
IRAS F14348-1447 & $2012-10$ & 20.5 & 309,231 & $63.4 \pm 1.9$ & $<4.2$ & 390.8 \\
CGCG 049-057 & $2011-09$ & 10.5 & 173,147 & $53.0 \pm 2.8$ & $<6.3$ & 788.4 \\
NGC 5936 & $2012-10$ & 61.5 & 253,179 & $120.1 \pm 1.1$ & $7.2 \pm 1.1$ & 488.8 \\
IRAS F16164-0746 & $2012-10$ & 10.0 & 176,122 & $155.8 \pm 1.7$ & $11.1 \pm 1.2$ & 308.8 \\
CGCG 052-037 & $2011-12$ & 10.5 & 240,152 & $104.9 \pm 3.2$ & $<5.7$ & 702.0 \\
IRAS F16399-0937 & $2012-10$ & 20.0 & 182,125 & $100.5 \pm 1.3$ & $5.2 \pm 1.3$ & 676.5 \\
NGC 6285 & $2011-12$ & 16.0 & 243,154 & $99.0 \pm 2.5$ & $4.7 \pm 1.2$ & 652.1 \\
NGC 6286 & $2011-12$ & 10.5 & 170,109 & $65.7 \pm 1.9$ & $<3.9$ & 698.9 \\
IRAS F17138-1017 & $2011-12$ & 10.5 & 216,116 & $270.4 \pm 3.0$ & $19.6 \pm 1.4$ & 957.3 \\
UGC 11041 & $2011-12$ & 10.5 & 245,141 & $174.7 \pm 2.8$ & $5.3 \pm 1.1$ & 542.6 \\
CGCG 141-034 & $2012-10$ & 10.0 & 196,148 & $161.5 \pm 2.3$ & $11.3 \pm 1.7$ & 542.1 \\
IRAS 18090+0130 & $2012-10$ & 10.0 & 171,118 & $53.3 \pm 2.6$ & $5.4 \pm 1.3$ & 699.6 \\
NGC 6701 & $2011-09$ & 16.0 & 186,137 & $113.1 \pm 2.0$ & $4.8 \pm 1.3$ & 722.5 \\
NGC 6786 & $2012-10$ & 10.5 & 211,155 & $202.3 \pm 2.1$ & $14.0 \pm 1.3$ & 308.8 \\
UGC 11415 & $2011-09$ & 10.5 & 193,134 & $77.2 \pm 2.2$ & $7.8 \pm 1.1$ & 479.9 \\
ESO 593-IG008 & $2011-09$ & 10.5 & 192,134 & $58.8 \pm 2.2$ & $<3.3$ & 506.6 \\
NGC 6907 & $2014-03$ & 20.5 & 190,147 & $112.5 \pm 2.5$ & $<5.1$ & 1172.0 \\
IRAS 21101+5810 & $2012-10$ & 15.5 & 367,245 & $278.4 \pm 4.4$ & $<11.1$ & 924.1 \\
ESO 602-G025 & $2011-09$ & 10.5 & 165,127 & $42.8 \pm 1.9$ & $<3.9$ & 648.5 \\
UGC 12150 & $2014-03$ & 25.5 & 251,162 & $134.4 \pm 2.0$ & $8.2 \pm 1.3$ & 729.0 \\
IRAS F22491-1808 & $2012-10$ & 15.5 & 247,156 & $77.3 \pm 2.7$ & $<4.2$ & 778.4 \\
CGCG 453-062 & $2014-03$ & 133.0 & 142,117 & $19.5 \pm 0.6$ & $<1.8$ & 520.2 \\
2MASX J23181352+0633267 & $2012-10$ & 15.5 & 293,177 & $60.4 \pm 3.0$ & $<3.6$ & 598.7 \\
\hline
\end{tabular}

Table 3. Derived parameters.

\begin{tabular}{|c|c|c|c|c|}
\hline Source name & $\begin{array}{c}L_{12}^{\prime} \mathrm{CO} \\
\left(10^{8} \mathrm{~K} \mathrm{~km} \mathrm{~s}^{-1} \mathrm{pc}^{2}\right) \\
(2)\end{array}$ & $\begin{array}{c}L_{13}^{\prime} \mathrm{CO} \\
\left(10^{8} \mathrm{~K} \mathrm{~km} \mathrm{~s}^{-1} \mathrm{pc}^{2}\right) \\
(3)\end{array}$ & (4) & $\begin{array}{c}M_{\mathrm{H}_{2}} \\
\left(10^{9} M_{\odot}\right) \\
(5)\end{array}$ \\
\hline NGC 0034 & $21.52 \pm 2.24$ & $<0.96$ & 0.0194 & $3.83 \pm 0.40$ \\
\hline Arp $256 \mathrm{~N}$ & $5.08 \pm 0.67$ & $1.74 \pm 0.46$ & 0.0271 & $0.90 \pm 0.12$ \\
\hline Arp $256 \mathrm{~S}$ & $15.35 \pm 1.63$ & $<1.68$ & 0.0271 & $2.73 \pm 0.29$ \\
\hline IC 1623 & $84.94 \pm 8.50$ & $2.23 \pm 0.31$ & 0.0200 & $15.12 \pm 1.51$ \\
\hline MCG -03-04-014 & $54.06 \pm 5.45$ & $<2.97$ & 0.0351 & $9.62 \pm 0.97$ \\
\hline IRAS F01364-1042 & $40.12 \pm 4.27$ & $<4.38$ & 0.0482 & $7.14 \pm 0.76$ \\
\hline IC 0214 & $26.11 \pm 2.71$ & $2.78 \pm 0.53$ & 0.0302 & $4.65 \pm 0.48$ \\
\hline UGC 01845 & $23.25 \pm 2.34$ & $1.42 \pm 0.25$ & 0.0157 & $4.14 \pm 0.42$ \\
\hline NGC 0958 & $24.34 \pm 2.54$ & $2.30 \pm 0.41$ & 0.0193 & $4.33 \pm 0.45$ \\
\hline ESO 550-IG025 & $33.10 \pm 3.55$ & $<1.98$ & 0.0321 & $5.89 \pm 0.63$ \\
\hline UGC 03094 & $35.66 \pm 3.65$ & $3.88 \pm 0.52$ & 0.0245 & $6.35 \pm 0.65$ \\
\hline NGC 1797 & $12.83 \pm 1.30$ & $0.83 \pm 0.14$ & 0.0150 & $2.28 \pm 0.23$ \\
\hline IRAS F05189-2524 & $26.08 \pm 2.64$ & $<1.26$ & 0.0428 & $4.64 \pm 0.47$ \\
\hline IRAS F05187-1017 & $23.24 \pm 2.33$ & $1.35 \pm 0.25$ & 0.0289 & $4.14 \pm 0.42$ \\
\hline IRAS F06076-2139 & $24.27 \pm 2.67$ & $<2.22$ & 0.0375 & $4.32 \pm 0.48$ \\
\hline NGC 2341 & $13.90 \pm 1.42$ & $1.01 \pm 0.18$ & 0.0171 & $2.47 \pm 0.25$ \\
\hline NGC 2342 & $15.59 \pm 1.58$ & $1.13 \pm 0.19$ & 0.0176 & $2.77 \pm 0.28$ \\
\hline IRAS 07251-0248 & $64.84 \pm 8.22$ & $<7.65$ & 0.0877 & $11.54 \pm 1.46$ \\
\hline IRAS F09111-1007 W & $52.43 \pm 5.46$ & $\ldots$ & 0.0543 & $9.33 \pm 0.97$ \\
\hline
\end{tabular}

Notes. (1) Source name; (2) ${ }^{12} \mathrm{CO}$ line luminosity; (3) ${ }^{13} \mathrm{CO}$ line luminosity; (4) Redshift obtained from the ${ }^{12} \mathrm{CO}$ line; (5) Molecular gas mass, derived using $\alpha=1.8 M_{\odot}\left(\mathrm{K} \mathrm{km} \mathrm{s}^{-1} \mathrm{pc}^{2}\right)^{-1}$ (see Sect. 5.1 for details). In those cases where the detection was not significant, the $3 \sigma$ upper limit is quoted. 
Table 3. continued.

\begin{tabular}{|c|c|c|c|c|}
\hline Source name & $\begin{array}{c}\left.L_{12}^{\prime} \mathrm{CO}^{-1} \mathrm{pc}^{2}\right) \\
\left(10^{8} \mathrm{~K} \mathrm{~km} \mathrm{~s}^{(2)}\right.\end{array}$ & $\begin{array}{c}\left.L_{13}^{\prime} \mathrm{CO}^{-1} \mathrm{pc}^{2}\right) \\
\left(10^{8} \mathrm{~K} \mathrm{~km} \mathrm{~s}^{(3)}\right.\end{array}$ & $\begin{array}{l}z \\
(4) \\
\end{array}$ & $\begin{array}{c}M_{\mathrm{H}_{2}} \\
\left(10^{9} M_{\odot}\right) \\
(5)\end{array}$ \\
\hline IRAS F09111-1007 E & $26.52 \pm 3.71$ & & 0.0547 & $4.72 \pm 0.66$ \\
\hline UGC 05101 & $61.37 \pm 6.23$ & $<1.68$ & 0.0393 & $10.92 \pm 1.11$ \\
\hline 2MASX J11210825-0259399 & & $\ldots$ & & \\
\hline CGCG 011-076 & $31.94 \pm 3.28$ & $2.61 \pm 0.76$ & 0.0248 & $5.69 \pm 0.58$ \\
\hline IRAS F12224-0624 & $6.33 \pm 0.69$ & $<0.84$ & 0.0264 & $1.13 \pm 0.12$ \\
\hline CGCG 043-099 & $46.61 \pm 4.74$ & $3.51 \pm 0.90$ & 0.0374 & $8.30 \pm 0.84$ \\
\hline ESO 507-G070 & $26.23 \pm 2.73$ & $<0.93$ & 0.0214 & $4.67 \pm 0.49$ \\
\hline NGC 5104 & $20.65 \pm 2.11$ & $1.60 \pm 0.37$ & 0.0186 & $3.68 \pm 0.38$ \\
\hline IC 4280 & $14.35 \pm 1.50$ & $1.13 \pm 0.23$ & 0.0163 & $2.55 \pm 0.27$ \\
\hline NGC 5258 & $31.08 \pm 3.20$ & $2.69 \pm 0.52$ & 0.0231 & $5.53 \pm 0.57$ \\
\hline UGC 08739 & $26.61 \pm 2.68$ & $2.05 \pm 0.25$ & 0.0168 & $4.74 \pm 0.48$ \\
\hline NGC 5331 & $59.89 \pm 6.12$ & $4.85 \pm 0.95$ & 0.0331 & $10.66 \pm 1.09$ \\
\hline CGCG 247-020 & $19.31 \pm 2.02$ & $<1.29$ & 0.0258 & $3.44 \pm 0.36$ \\
\hline IRAS F14348-1447 & $169.52 \pm 19.23$ & $<20.64$ & 0.0826 & $30.17 \pm 3.42$ \\
\hline CGCG 049-057 & $9.21 \pm 0.92$ & $0.63 \pm 0.10$ & 0.0130 & $1.64 \pm 0.16$ \\
\hline NGC 5936 & $12.55 \pm 1.26$ & $1.00 \pm 0.13$ & 0.0133 & $2.23 \pm 0.22$ \\
\hline IRAS F16164-0746 & $26.72 \pm 2.80$ & $<1.50$ & 0.0237 & $4.76 \pm 0.50$ \\
\hline CGCG 052-037 & $27.45 \pm 2.77$ & $1.61 \pm 0.38$ & 0.0245 & $4.89 \pm 0.49$ \\
\hline IRAS F16399-0937 & $32.98 \pm 3.40$ & $1.77 \pm 0.44$ & 0.0270 & $5.87 \pm 0.61$ \\
\hline NGC 6285 & $10.91 \pm 1.14$ & $<0.63$ & 0.0191 & $1.94 \pm 0.20$ \\
\hline NGC 6286 & $43.05 \pm 4.33$ & $3.51 \pm 0.38$ & 0.0187 & $7.66 \pm 0.77$ \\
\hline IRAS F17138-1017 & $23.72 \pm 2.40$ & $0.81 \pm 0.17$ & 0.0173 & $4.22 \pm 0.43$ \\
\hline UGC 11041 & $19.33 \pm 1.95$ & $1.52 \pm 0.24$ & 0.0163 & $3.44 \pm 0.35$ \\
\hline CGCG 141-034 & $9.80 \pm 1.09$ & $1.12 \pm 0.26$ & 0.0201 & $1.74 \pm 0.19$ \\
\hline IRAS $18090+0130$ & $42.98 \pm 4.36$ & $2.06 \pm 0.54$ & 0.0289 & $7.65 \pm 0.78$ \\
\hline NGC 6701 & $15.84 \pm 1.59$ & $1.24 \pm 0.15$ & 0.0132 & $2.82 \pm 0.28$ \\
\hline NGC 6786 & $22.15 \pm 2.30$ & $2.50 \pm 0.39$ & 0.0251 & $3.94 \pm 0.41$ \\
\hline UGC 11415 & $17.17 \pm 1.84$ & $<0.99$ & 0.0253 & $3.06 \pm 0.33$ \\
\hline ESO 593-IG008 & $123.62 \pm 12.67$ & $<5.82$ & 0.0488 & $22.00 \pm 2.26$ \\
\hline NGC 6907 & $14.17 \pm 1.43$ & $<0.57$ & 0.0106 & $2.52 \pm 0.26$ \\
\hline IRAS $21101+5810$ & $30.17 \pm 3.30$ & $<2.67$ & 0.0392 & $5.37 \pm 0.59$ \\
\hline ESO 602-G025 & $38.77 \pm 3.92$ & $2.66 \pm 0.45$ & 0.0252 & $6.90 \pm 0.70$ \\
\hline UGC 12150 & $16.27 \pm 1.72$ & $<0.87$ & 0.0215 & $2.90 \pm 0.31$ \\
\hline IRAS F22491-1808 & $55.12 \pm 5.74$ & $<4.86$ & 0.0777 & $9.81 \pm 1.02$ \\
\hline CGCG 453-062 & $17.01 \pm 1.90$ & $<1.02$ & 0.0249 & $3.03 \pm 0.34$ \\
\hline 2MASX J23181352+0633267 & $\ldots$ & $\ldots$ & $\ldots$ & $\ldots$ \\
\hline
\end{tabular}

is compatible with our results. On the other hand, their IRAS F05189-2524 and IRAS F14348-1447 measurements $\left((47.9 \pm 9.6) \times 10^{8}\right.$ and $(123.0 \pm 24.6) \times 10^{8} \mathrm{~K} \mathrm{~km} \mathrm{~s}^{-1} \mathrm{pc}^{2}$, respectively), although significantly higher, most likely due to extended emission and the larger FWHM of the NRAO $12 \mathrm{~m}$ telescope ( 55 arcsec), are also compatible with our result within $2 \sigma$. To take the extended emission into account, we have performed aperture photometry as detailed in Sect. 5.1.

We obtained the molecular gas mass $\left(M_{\mathrm{H}_{2}}\right)$ from the integrated $\mathrm{CO}$ intensities assuming a constant CO-to- $\mathrm{H}_{2}$ conversion factor, $\alpha=1.8 M_{\odot}\left(\mathrm{K} \mathrm{km} \mathrm{s}^{-1} \mathrm{pc}^{2}\right)^{-1}$. The derivation of this factor is also explained in detail in Sect. 5.1.

\section{Discussion}

The relationship between the molecular gas content and the star formation properties (rate, efficiency, or depletion time) has been thoroughly studied (e.g., Leroy et al. 2008; Daddi et al. 2010; Genzel et al. 2010). Our study allows us to constrain several of these correlations with a large and uniformly observed sample.
However, to that end we first need to investigate whether the conversion between $\mathrm{CO}$ and $\mathrm{H}_{2}, \alpha$, is identical for local spiral galaxies and (U)LIRGs, as is often assumed, and determine its value.

\subsection{Gas-to-dust ratio and determination of $\alpha$}

There are extensive discussions in the literature over the proper value of $\alpha$ to use when deriving molecular gas masses of (U)LIRGs (e.g., Solomon et al. 1997; Downes \& Solomon 1998; Yao et al. 2003; Papadopoulos et al. 2012b; Bolatto et al. 2013). Prior to 1997, the standard was to make use of the value of $\alpha$ derived for the Milky Way, $\alpha_{\mathrm{MW}} \simeq 4 M_{\odot}\left(\mathrm{K} \mathrm{km} \mathrm{s}^{-1} \mathrm{pc}^{2}\right)^{-1}$, with the reasoning that the molecular gas was bound in physically distinct, virialized clouds, in which increases (decreases) in the temperature of the gas, $T$, were offset by decreases (increases) in the gas density, $\rho$; that is,

$M_{\text {cloud }} \propto\left(\rho^{1 / 2} / T\right) L_{\mathrm{CO}}^{\prime}=($ constant $) \times L_{\mathrm{CO}}^{\prime}$

(e.g., Scoville \& Sanders 1987). However, arguments based on estimates of dynamical masses in (U)LIRGs led to the 
hypothesis that $\alpha_{\mathrm{MW}}$ was too high for this type of object. That is, the dynamical masses of (U)LIRGs as traced by CO kinematics were lower than the gas masses derived using $\alpha_{\mathrm{MW}}-$ and therefore the value of $\alpha$ in these extreme starburst galaxies should be at least proportionally lower (e.g., Downes \& Solomon 1998). It was speculated that a primary difference between normal spiral galaxies and (U)LIRGs is that the "inter-cloud" medium regions of the latter population likely contain significant amounts of molecular gas (Solomon et al. 1997); this means that the molecular gas distribution is more uniform within the starburst region, invalidating the Milky Way assumption of discrete molecular clouds.

To address this issue, in this paper we assume that (i) the relative mass fraction of atomic and molecular hydrogen is similar among our galaxies $\left(M_{\mathrm{HI}} / M_{\mathrm{H}_{2}}=\gamma\right)$, and (ii) any observed offset is driven by the physics setting the value of $\alpha$ for each population. Therefore, we assume that

$\frac{M_{\mathrm{gas}}}{M_{\mathrm{dust}}}=\frac{(1+\gamma) \alpha_{\mathrm{n}} L_{\mathrm{CO}}^{\prime}}{M_{\text {dust }}}($ normal $)=\frac{(1+\gamma) \alpha_{\mathrm{L}} L_{\mathrm{CO}}^{\prime}}{M_{\text {dust }}}(\mathrm{LIRG})$,

where $n$ and $L$ subscripts are used for normal and LIRGs, respectively (see Bolatto et al. 2013, for an extensive discussion of dustbased conversion factor determinations). To proceed, we used for comparison the Key Insights on Nearby Galaxies: a FarInfrared Survey with Herschel (KINGFISH) ${ }^{4}$ sample of local spiral galaxies observed with Herschel by Dale et al. (2012) that have pre-existing CO measurements in two surveys: the FCRAO extragalactic CO survey (Young et al. 1995), with ${ }^{12} \mathrm{CO}(1-0)$ measurements, and the HERACLES survey from Leroy et al. (2009), with ${ }^{12} \mathrm{CO}(2-1)$ observations, for which we have used a conversion factor of $R_{21}=0.8$ for the conversion between ${ }^{12} \mathrm{CO}(2-1)$ and ${ }^{12} \mathrm{CO}(1-0)$ as suggested by the authors (Leroy et al. 2009). Both sets of data encompass most of the galaxies' emission. In particular, the FCRAO sample was observed with a $14 \mathrm{~m}$ dish (i.e., FWHM as large as $\simeq 45$ arcsec), while the HERACLES galaxies were spatially mapped with the Heterodyne Receiver Array (HERA) multi-pixel receiver on the IRAM-30 m telescope.

There is a correlation between the gas-to-dust ratio and both the stellar mass (Cortese et al. 2016) and metallicity (e.g., Rémy-Ruyer et al. 2014). These two correlations are likely linked through the mass-metallicity relation (Tremonti et al. 2004). To check whether we expect a variation of gas-to-dust ratios among our samples, we compared $L_{\mathrm{CO}}^{\prime} / M_{\text {dust }}$ with the Two Micron All-Sky Survey (2MASS) H-band luminosity, which is a first order tracer of the stellar mass of galaxies. As shown in Fig. 3, we do not find any trend, either in our sample or in the comparison FCRAO or HERACLES sources, so no stellar mass correction was applied to our derived gas-to-dust ratios. The reason why a clear dependency is not seen in the figure is likely to be in the mass-metallicity relation itself: while we expect a difference in metallicity of $\sim 0.1$ dex for a stellar mass variation of two orders of magnitude (the range of GOALS galaxies is $9.5<$ $\log \left(M_{\star}\right)<11.5$ ) (Torrey et al. 2018), there is a large scatter between the two magnitudes, of approximately $\sim 0.2-0.3 \mathrm{dex}$. This scatter is the cause of why we do not see variations with the gas-to-dust ratio over the traced range of stellar masses. From Fig. 3 it is also noticeable that two sources from the FCRAO comparison sample (NGC 3077 and NGC 4569) have significantly lower $H$-band luminosities than any other source in this study. These were excluded from the analysis below, as they are not representative of the global characteristics of both populations.

\footnotetext{
4 https://www.ast.cam.ac.uk/research/kingfish
}

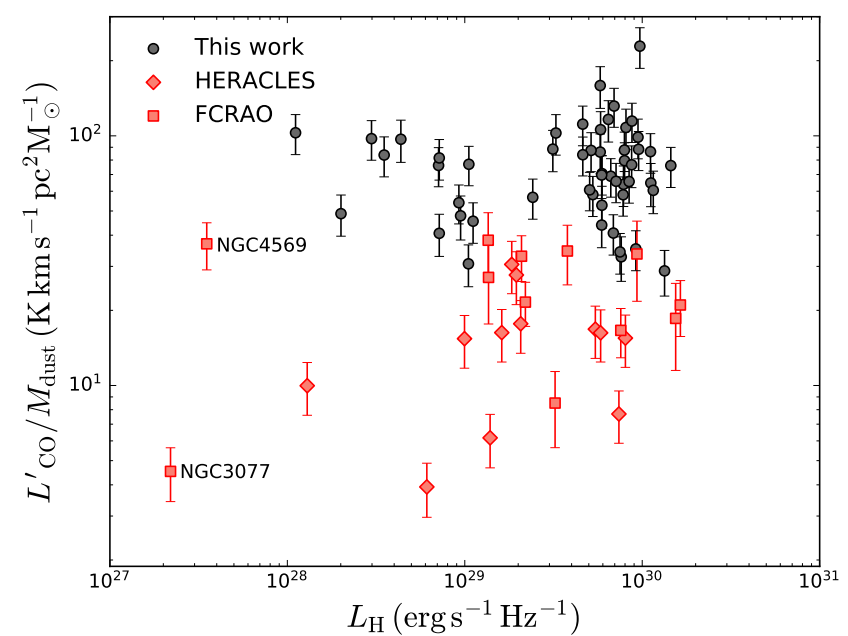

Fig. 3. Ratio between the CO luminosity and the dust mass as a function of the rest-frame $H$-band luminosity, tracer of stellar mass. There is no obvious trend in our sample (in black) or in the local comparison samples (in red). Given the low $H$-band luminosity of NGC 3077 and NGC 4569, these were excluded from the comparison. Details are given in the main text.

To obtain the dust properties of the galaxies, both for our sources and for the local comparison samples, we constructed far infrared (FIR) spectral energy distributions (SEDs) using the new Herschel photometric catalog by (Chu et al. 2017) between 70 and $350 \mu \mathrm{m}$ in order to perform a model fitting. Although the angular resolution of the IRAM-30 m telescope $\left(\sim 22^{\prime \prime}\right)$ is generally sufficient to encompass most of the FIR extension of each galaxy, this is not always the case, as shown in the left panels of the molecular gas observations of Figs. 2 and A.1, where we have plotted the IRAM beam sizes over the Herschel $70 \mu \mathrm{m}$ images. In order to compare gas (derived from $\mathrm{CO}$ ) and dust (derived from FIR) properties, it is essential to consider the same apertures for both datasets. To ensure this, we convolved our Herschel images to the angular resolution of our IRAM-30 m data using Gaussian smoothing, and performed aperture photometry centered in the IRAM-30 m pointing position. We did not smooth our Herschel Spectral and Photometric Imaging Receiver (SPIRE) $350 \mu \mathrm{m}$ images, which already have a resolution $\left(\sim 25^{\prime \prime}\right)$ comparable with the ${ }^{12} \mathrm{CO}$ measurements. We excluded our $500 \mu \mathrm{m}$ images due to their coarser resolution than the IRAM-30 m beam.

We then fit each SED to an optically thin modified blackbody to derive the dust temperatures, $T_{\text {dust }}$, and masses, $M_{\text {dust }}$ for each galaxy (see Appendix B). To calculate the dust masses, Scoville et al. (2016) argue for the use of a common massweighted dust temperature for all sources $\left(T_{\text {dust }}=25 \mathrm{~K}\right)$, instead of that obtained from their individual SED fits, which is more representative from localized warm, luminous regions. In this paper we follow this strategy, so we note that any comparison between samples must take this difference into account. For our sample, the use of a fixed $T_{\text {dust }}=25 \mathrm{~K}$ yields an average $M_{\text {dust }}$ 1.53 times higher than when a SED fitted temperature is used.

By integrating below the fitted blackbody we directly obtain the luminosity between 42.5 and $122.5 \mu \mathrm{m}, L_{\text {FIR }}$. Additionally, we also calculate the total infrared (TIR) luminosity (between 3 and $\left.1100 \mu \mathrm{m}, L_{\mathrm{TIR}}\right)$, making use of the prescription provided by Dale \& Helou (2002):

$$
\begin{aligned}
L_{\mathrm{TIR}}= & 1.559 v L_{v}(24 \mu \mathrm{m})+0.7686 v L_{v}(70 \mu \mathrm{m}) \\
& +1.347 v L_{v}(160 \mu \mathrm{m}),
\end{aligned}
$$


using the Spitzer Multiband Imaging Photometer (MIPS) $24 \mu \mathrm{m}$ data from Mazzarella et al. (in prep.) for our galaxies (after applying the same Gaussian smoothing described above), and the local sample data from Dale et al. (2007, 2009) and Engelbracht et al. (2008). We note that the TIR luminosities are obtained from galaxy-integrated photometry, and thus are not matched to the IRAM beam. All fitted parameters (plus $L_{\mathrm{TIR}}$ ) are presented in Table 4. For completeness, we provide as well the galaxy-integrated parameters obtained by using the total flux for each filter (including SPIRE $500 \mu \mathrm{m}$ in this case). Unless specifically mentioned otherwise, throughout this paper we always refer to or use the parameters obtained from the SED fitting to the aperture-matched photometry. For details on the modified blackbody SED fitting, we refer the reader to Appendix B.

In Fig. 4 we show the gas-to-dust mass ratio $\left(M_{\mathrm{H}_{2}} / M_{\text {dust }}\right)$ for both our (U)LIRGs and the local control sample (FCRAO + HERACLES) as a function of the star formation rate (SFR) surface density $\left(\Sigma_{\mathrm{SFR}}\right.$; obtained from Leroy et al. 2013 and Díaz-Santos et al. 2017), showing that the two populations have distinctly different $\Sigma_{\text {SFR }}$ and thus physical conditions (e.g., Díaz-Santos et al. 2017). The top panels of the figure show the gas-to-dust mass ratios obtained by deriving the dust masses based on the fitted $250 \mu \mathrm{m}$ continuum, using a mass absorption coefficient of $\kappa(250 \mu \mathrm{m})=0.48 \mathrm{~m}^{2} \mathrm{~kg}^{-1}$ (see Appendix B for details) and by leaving $T_{\text {dust }}$ and $\beta$ as free parameters. The middle panels are the same as the top, but for a fixed emissivity index of $\beta=1.8$ (Planck Collaboration XXI 2011). Finally, the bottom panels show the gas-to-dust mass ratios obtained by fixing $\beta$ and assuming a fixed $25 \mathrm{~K}$ dust temperature for every source, as proposed by Scoville et al. (2016). For the remainder of the discussion we have adopted the results from the latter method, while we occasionally refer to the results obtained when letting $T_{\text {dust }}$ free.

If a Milky Way $\alpha$ is adopted for the reference sample of normal star-forming galaxies, we find that in order to match the offset between the median gas-to-dust ratio of (U)LIRGs relative to the reference sample, within their median absolute deviations, it is necessary to adopt a value $\alpha_{(\mathrm{U}) \mathrm{LIRG}}=1.8_{-0.8}^{+1.3} M_{\odot}\left(\mathrm{K} \mathrm{km} \mathrm{s}^{-1} \mathrm{pc}^{2}\right)^{-1}$, assuming a fixed $T_{\text {dust }}$. If we instead use the dust temperatures from SED fitting to derive the dust masses, we require $\alpha_{(\mathrm{U}) \mathrm{LIRG}}=$ $0.9_{-0.5}^{+0.9}$ in order to match the gas-to-dust ratios. Our derived value of $\alpha$ is compatible with the values derived by Downes \& Solomon (1998, $\alpha=0.8$ ), Papadopoulos et al. (2012b, $\alpha=0.6 \pm 0.2)$, and Scoville et al. (2015) for the (U)LIRG Arp $220(\alpha \sim 2)$.

\subsection{Impact of varying ISM assumptions}

We stress that our derived value of $\alpha$ strongly depends on two assumptions: a constant gas-to-dust ratio and a constant relative fraction of atomic to molecular hydrogen gas, $\gamma$. Some studies have claimed evidence of the gas-to-dust ratio of (U)LIRGs being compatible with that of normal galaxies (see, e.g., Wilson et al. 2008). However, these ratios are calibrated based on a lower value of $\alpha$ for (U)LIRGs, falling in a circular argument.

In any case, even if either of the two ratios is proven to be different in LIRGs, the following relation derived from Eq. (4) and the offset between samples seen in Fig. 4 should still hold:

$\alpha_{\mathrm{L}}=\left(0.45_{-0.2}^{+0.3}\right) \alpha_{\mathrm{n}}\left(\frac{1+\gamma_{\mathrm{n}}}{1+\gamma_{\mathrm{L}}}\right)\left(\frac{L_{\mathrm{CO}}^{\prime}}{M_{\text {dust }}}\right)_{\mathrm{n}}\left(\frac{L_{\mathrm{CO}}^{\prime}}{M_{\text {dust }}}\right)_{\mathrm{L}}^{-1}$.

We note that if the assumption is made that the gas-to-dust mass ratio and $\alpha$ are constant, but instead we vary the relative fraction of atomic to molecular gas between the two samples, the end result would be a decrease in $\gamma$ for the (U)LIRGs. In particular, we would get $\left(1+\gamma_{\mathrm{n}}\right) /\left(1+\gamma_{\mathrm{L}}\right)=2.2$. This equation yields unphysical negative $\gamma_{\mathrm{L}}$ values for any $\gamma_{\mathrm{n}}$ value below 1.2. The observed difference in Fig. 4 between the two is unlikely thus to be ascribed solely to a different $\gamma$.

\subsection{Star formation efficiencies and gas depletion timescales}

Figure 5 shows the FIR (derived from the modified blackbody SED fits) and the TIR (obtained from Eq. (5)) luminosities as a function of the $L_{\mathrm{CO}}^{\prime}$ for our sample of GOALS galaxies and for the local comparison samples. In optically thick environments, and in the absence of significant dust heating by an active galactic nucleus (AGN), the plot of $L_{\mathrm{TIR}, \mathrm{FIR}}$ versus $L_{\mathrm{CO}}^{\prime}$ is essentially a comparison between the energy generated by the embedded starburst(s) versus the total reservoir of gas available to form new stars.

For the purpose of fitting the data, we excluded (U)LIRGs where an AGN contributes $>20 \%$ to the energy output, where we used the AGN bolometric fractions obtained from the combination of several mid-IR diagnostics (Díaz-Santos et al. 2017). The reason behind this exclusion is to avoid contamination in the estimation of the star formation rates, efficiencies, and gas depletion times, as strong AGN can contribute significantly to the FIR luminosity. These sources were not excluded from the analysis in Sect. 5.1 because there is not a known dependence of gas-to-dust mass ratio with AGN fraction, and our size sample does not allow us to study possible effects on it. No sources were removed from the local comparison sample, which is not dominated by AGN by design.

Three sources were discarded based on the above AGN criterion: NGC 0958, UGC 05101, and IRAS F05189-2524. Furthermore, to avoid the instability of ordinary least-squares fits when both dependent and independent variables have an associated error, we followed the same approach as Greve et al. (2014) and used a Bayesian method to fit the data. Specifically, we used Josh Mayer's Python port ${ }^{5}$ of the LINMIX_ERR IDL package (Kelly 2007).

The combined fit (our (U)LIRG sample and the local, normal, star-forming sources) to the data in Fig. 5 has the functional form

$\log \left(L_{\mathrm{TIR}}\right)=(0.97 \pm 0.06) \log \left(L_{\mathrm{CO}}^{\prime}\right)+(1.75 \pm 0.51)$
$\log \left(L_{\mathrm{FIR}}\right)=(1.05 \pm 0.06) \log \left(L_{\mathrm{CO}}^{\prime}\right)+(0.73 \pm 0.58)$,

with median Pearson correlation coefficients of $\rho=0.923$ and $\rho=0.915$, respectively, and a robust statistical significance ( $p$-values of $4.1 \times 10^{-28}$ and $5.0 \times 10^{-26}$, respectivey). We note that $L_{\mathrm{TIR}}$ is derived from integrated-galaxy photometry (see Eq. (5)), while $L_{\mathrm{FIR}}$ is obtained from integrating the fit to the aperturematched SEDs. More luminous galaxies, with warmer dust, will have a larger contribution to the emission from shorter wavelengths (our fit uses $70<\lambda / \mu \mathrm{m}<350$ ), and thus $L_{\text {FIR }}$ could be underestimated for those sources. We find, however, that there is no significant difference in the use of $L_{\mathrm{TIR}}$ or $L_{\mathrm{FIR}}$ for our sample. By comparison, Greve et al. (2014) derived a linear value for the slope of $(1.00 \pm 0.05)$ and $(0.99 \pm 0.04)$ for $L_{(8-1000 \mu \mathrm{m})}$ and $L_{(50-300 \mu \mathrm{m})}$, respectively. Their luminosity dynamic range was $10.29 \leq \log \left(L_{(8-1000 \mu \mathrm{m})}\right) \leq 12.56$. We also note that their sample included high- $z$ galaxies which, if they had not been included, would have caused the slope to be sub-linear. We only find a very slight steepening in the slope of the fit to the (U)LIRGs with respect to the local comparison sample, but not as pronounced

https://github.com/jmeyers314/linmix/ 
Table 4. Derived dust parameters and luminosities for our (U)LIRG sample.

\begin{tabular}{|c|c|c|c|c|c|c|c|c|}
\hline \multirow[b]{2}{*}{ Source name } & \multicolumn{3}{|c|}{ Overall SED fit } & \multicolumn{5}{|c|}{ Within IRAM-30 $\mathrm{m}$ beam } \\
\hline & $\begin{array}{l}T_{\text {dust }} \\
(\mathrm{K}) \\
\end{array}$ & $\begin{array}{c}M_{\text {dust }}^{\text {fit }} \\
\left(10^{7} M_{\odot}\right)\end{array}$ & $\begin{array}{c}\log \left(L_{\mathrm{FIR}}\right) \\
\left(L_{\odot}\right)\end{array}$ & $\begin{array}{l}T_{\text {dust }} \\
(\mathrm{K})\end{array}$ & $\begin{array}{c}M_{\text {dust }}^{\text {fit }} \\
\left(10^{7} M_{\odot}\right)\end{array}$ & $\begin{array}{c}M_{\text {dust }}^{25 \mathrm{~K}} \\
\left(10^{7} M_{\odot}\right)\end{array}$ & $\begin{array}{c}\log \left(L_{\mathrm{FIR}}\right) \\
\left(L_{\odot}\right)\end{array}$ & $\begin{array}{c}\log \left(L_{\mathrm{TIR}}\right) \\
\left(L_{\odot}\right)\end{array}$ \\
\hline NGC 0034 & $34.87 \pm 0.59$ & $4.11_{-0.40}^{+0.42}$ & $11.15_{-0.08}^{+0.07}$ & $36.32 \pm 1.08$ & $1.35_{-0.22}^{+0.23}$ & $3.17_{-0.50}^{+0.55}$ & $10.77_{-0.13}^{+0.12}$ & 11.02 \\
\hline Arp $256 \mathrm{~N}$ & $25.65 \pm 2.14$ & $4.18_{-2.13}^{+2.87}$ & $10.29_{-0.50}^{+0.37}$ & $26.04 \pm 0.51$ & $1.25_{-0.17}^{+0.19}$ & $1.38_{-0.19}^{+0.21}$ & $9.81_{-0.12}^{+0.09}$ & 10.12 \\
\hline Arp 256S & $31.50 \pm 0.64$ & $6.11_{-0.83}^{+0.89}$ & $11.04_{-0.11}^{+0.09}$ & $33.22 \pm 0.87$ & $1.88_{-0.29}^{+0.31}$ & $3.68_{-0.56}^{+0.60}$ & $10.67_{-0.12}^{+0.11}$ & 10.99 \\
\hline IC 1623 & $30.60 \pm 0.58$ & $13.72_{-1.25}^{+1.31}$ & $11.32_{-0.09}^{+0.07}$ & $32.11 \pm 0.85$ & $3.71_{-0.58}^{+0.63}$ & $6.73_{-1.05}^{+1.14}$ & $10.89_{-0.13}^{+0.11}$ & 11.21 \\
\hline MCG -03-04-014 & $30.14 \pm 0.56$ & $15.29_{-1.78}^{+1.89}$ & $11.32_{-0.10}^{+0.07}$ & $30.68 \pm 0.74$ & $6.26_{-0.94}^{+1.01}$ & $10.31_{-1.55}^{+1.67}$ & $10.98_{-0.12}^{+0.10}$ & 11.27 \\
\hline IRAS F01364-1042 & $34.92 \pm 0.82$ & $10.50_{-1.42}^{+1.52}$ & $11.54_{-0.11}^{-0.10}$ & $35.89 \pm 1.06$ & $4.14_{-0.66}^{-0.94}$ & $9.63_{-1.54}^{+1.67}$ & $11.20_{-0.13}^{+0.12}$ & 11.37 \\
\hline IC 0214 & $28.09 \pm 0.85$ & $11.72_{-2.27}^{+2.51}$ & $11.00_{-0.16}^{+0.13}$ & $29.84 \pm 0.69$ & $3.70_{-0.54}^{+0.00}$ & $5.71_{-0.84}^{-1.54}$ & $10.67_{-0.12}^{+0.13}$ & 10.96 \\
\hline UGC 01845 & $29.54 \pm 0.41$ & $5.40_{-0.50}^{+0.52}$ & $10.82_{-0.07}^{+0.06}$ & $29.92 \pm 0.72$ & $2.09_{-0.31}^{+0.34}$ & $3.23_{-0.48}^{+0.52}$ & $10.44_{-0.12}^{+0.10}$ & 10.73 \\
\hline NGC 0958 & $22.96 \pm 0.78$ & $30.26_{-6.27}^{+7.08}$ & $10.81_{-0.18}^{+0.015}$ & $23.86 \pm 0.45$ & $4.51_{-0.65}^{+0.71}$ & $3.99_{-0.57}^{+0.46}$ & $10.11_{-0.12}^{+0.12}$ & 10.50 \\
\hline ESO 550-IG025 & $28.79 \pm 0.35$ & $9.23_{-0.81}^{+0.84}$ & $10.97_{-0.07}^{-0.18}$ & $28.19 \pm 0.64$ & $5.18_{-0.78}^{+0.03}$ & $7.00_{-1.05}^{+1.14}$ & $10.66_{-0.13}^{+0.12}$ & 10.96 \\
\hline UGC 03094 & $26.39 \pm 0.59$ & $18.25_{-2.62}^{+2.82}$ & $11.02_{-0.12}^{+0.09}$ & $27.23 \pm 0.60$ & $5.43_{-0.81}^{+0.88}$ & $6.74_{-1.01}^{+1.09}$ & $10.58_{-0.12}^{+0.10}$ & 10.91 \\
\hline NGC 1797 & $29.05 \pm 0.56$ & $4.23_{-0.52}^{+0.55}$ & $10.67_{-0.10}^{+0.08}$ & $30.79 \pm 0.76$ & $1.25_{-0.19}^{+0.20}$ & $2.06_{-0.31}^{+0.34}$ & $10.30_{-0.12}^{+0.11}$ & 10.62 \\
\hline IRAS F05189-2524 & $37.28 \pm 0.76$ & $10.49_{-1.20}^{+1.27}$ & $11.70_{-0.09}^{+0.08}$ & $37.63 \pm 1.19$ & $4.31_{-0.71}^{+0.77}$ & $11.01_{-1.82}^{+1.98}$ & $11.34_{-0.13}^{+0.12}$ & 11.69 \\
\hline IRAS F05187-1017 & $28.98 \pm 0.61$ & $10.10_{-1.31}^{+1.40}$ & $11.03_{-0.11}^{+0.09}$ & $29.46 \pm 0.71$ & $4.10_{-0.63}^{+0.68}$ & $6.14_{-0.94}^{+1.01}$ & $10.68_{-0.13}^{+0.10}$ & 10.91 \\
\hline IRAS F06076-2139 & $30.95 \pm 0.83$ & $12.17_{-1.97}^{+2.14}$ & $11.29_{-0.14}^{+0.11}$ & $32.09 \pm 0.85$ & $4.60_{-0.72}^{+0.78}$ & $8.39_{-1.31}^{+1.42}$ & $10.96_{-0.13}^{+0.11}$ & 11.22 \\
\hline NGC 2341 & $28.58 \pm 0.52$ & $5.50_{-0.63}^{+0.67}$ & $10.73_{-0.09}^{+0.08}$ & $30.20 \pm 0.72$ & $1.43_{-0.21}^{+0.23}$ & $2.26_{-0.34}^{+0.36}$ & $10.30_{-0.12}^{+0.10}$ & 10.59 \\
\hline NGC 2342 & $26.77 \pm 0.64$ & $10.50_{-1.49}^{+1.61}$ & $10.82_{-0.12}^{+0.10}$ & $28.23 \pm 0.64$ & $1.78_{-0.26}^{+0.28}$ & $2.40_{-0.36}^{+0.38}$ & $10.21_{-0.12}^{+0.10}$ & 10.55 \\
\hline IRAS 07251-0248 & $33.71 \pm 0.81$ & $45.47_{-6.38}^{+6.49}$ & $12.05_{-0.12}^{+0.12}$ & $33.28 \pm 0.95$ & $22.53_{-3.70}^{+4.03}$ & $45.51_{-7.47}^{+8.14}$ & $11.71_{-0.15}^{+0.11}$ & 11.96 \\
\hline IRAS F09111-1007 W & $30.30 \pm 0.55$ & $35.48_{-4.49}^{+4.50}$ & $11.68_{-0.11}^{+0.12}$ & $29.77 \pm 0.72$ & $15.29_{-2.34}^{+2.54}$ & $23.66_{-3.63}^{+3.41}$ & $11.27_{-0.13}^{+0.13}$ & 11.52 \\
\hline IRAS F09111-1007 E & $27.79 \pm 0.78$ & $16.79_{-3.22}^{+3.55}$ & $11.11_{-0.17}^{+0.11}$ & $26.55 \pm 0.55$ & $8.09_{-1.18}^{+1.28}$ & $9.47_{-1.39}^{+1.49}$ & $10.66_{-0.14}^{+0.08}$ & 11.01 \\
\hline UGC 05101 & $29.49 \pm 0.42$ & $47.76_{-4.29}^{+4.49}$ & $11.74_{-0.08}^{+0.17}$ & $29.96 \pm 0.74$ & $17.41_{-2.63}^{+2.85}$ & $27.21_{-4.11}^{+4.45}$ & $11.35_{-0.13}^{+0.14}$ & 11.61 \\
\hline 2MASX J11210825-0259399 & $22.94 \pm 2.60$ & $1.65_{-0.90}^{+1.32}$ & $9.54_{-0.61}^{+0.45}$ & $25.06 \pm 0.49$ & $0.53_{-0.08}^{+0.08}$ & $0.53_{-0.08}^{+0.08}$ & $9.32_{-0.12}^{+0.09}$ & 9.67 \\
\hline CGCG 011-076 & $27.01 \pm 0.52$ & $13.98_{-1.71}^{+1.82}$ & $10.97_{-0.11}^{+0.08}$ & $28.23 \pm 0.65$ & $3.81_{-0.57}^{+0.62}$ & $5.16_{-0.78}^{+0.84}$ & $10.53_{-0.12}^{+0.10}$ & 10.88 \\
\hline IRAS F12224-0624 & $33.46 \pm 0.67$ & $46.70_{-5.70}^{+6.05}$ & $12.05_{-0.10}^{+0.08}$ & $32.09 \pm 0.85$ & $2.06_{-0.32}^{+0.35}$ & $3.74_{-0.58}^{+0.63}$ & $10.62_{-0.13}^{+0.11}$ & 10.82 \\
\hline CGCG 043-099 & $28.89 \pm 0.54$ & $21.17_{-2.57}^{+2.73}$ & $11.33_{-0.10}^{+0.07}$ & $30.16 \pm 0.73$ & $5.41_{-0.82}^{+0.88}$ & $8.58_{-1.29}^{+1.40}$ & $10.86_{-0.13}^{+0.10}$ & 11.12 \\
\hline ESO 507-G070 & $33.06 \pm 0.61$ & $7.91_{-0.89}^{+0.94}$ & $11.29_{-0.09}^{+0.10}$ & $33.67 \pm 0.93$ & $2.25_{-0.35}^{+0.02}$ & $4.53_{-0.71}^{+0.77}$ & $10.79_{-0.13}^{+0.11}$ & 11.00 \\
\hline NGC 5104 & $27.07 \pm 0.43$ & $12.25_{-1.28}^{+1.35}$ & $10.92_{-0.09}^{+0.07}$ & $27.75 \pm 0.61$ & $3.14_{-0.46}^{+0.50}$ & $4.08_{-0.60}^{+0.65}$ & $10.40_{-0.12}^{+0.10}$ & 10.72 \\
\hline IC 4280 & $25.62 \pm 0.47$ & $12.86_{-1.56}^{+1.66}$ & $10.78_{-0.10}^{+0.08}$ & $26.82 \pm 0.57$ & $2.47_{-0.36}^{+0.39}$ & $2.95_{-0.43}^{+0.00}$ & $10.20_{-0.12}^{+0.10}$ & 10.53 \\
\hline NGC 5258 & $26.14 \pm 0.50$ & $18.35_{-2.15}^{+2.29}$ & $10.99_{-0.10}^{+0.10}$ & $27.59 \pm 0.59$ & $3.52_{-0.51}^{+0.55}$ & $4.51_{-0.66}^{+0.71}$ & $10.43_{-0.12}^{+0.09}$ & 10.75 \\
\hline UGC 08739 & $24.07 \pm 0.56$ & $20.62_{-3.03}^{+3.29}$ & $10.79_{-0.13}^{+0.10}$ & $24.95 \pm 0.49$ & $4.38_{-0.63}^{+0.68}$ & $4.36_{-0.63}^{+0.67}$ & $10.23_{-0.12}^{+0.09}$ & 10.57 \\
\hline NGC 5331 & $29.33 \pm 0.87$ & $16.55_{-3.90}^{+4.34}$ & $11.27_{-0.18}^{+0.14}$ & $28.15 \pm 0.62$ & $7.87_{-1.15}^{+1.24}$ & $10.60_{-1.55}^{+1.67}$ & $10.83_{-0.12}^{+0.09}$ & 11.12 \\
\hline CGCG 247-020 & $32.02 \pm 0.72$ & $5.11_{-0.70}^{+0.75}$ & $11.01_{-0.11}^{+0.10}$ & $32.52 \pm 0.84$ & $1.88_{-0.29}^{+0.31}$ & $3.51_{-0.54}^{+0.58}$ & $10.62_{-0.12}^{+0.11}$ & 10.91 \\
\hline IRAS F14348-1447 & $34.02 \pm 0.61$ & $26.38_{-2.72}^{+2.87}$ & $11.85_{-0.09}^{+0.07}$ & $32.71 \pm 0.90$ & $26.18_{-4.21}^{+4.57}$ & $50.76_{-8.15}^{+8.86}$ & $11.74_{-0.14}^{+0.10}$ & 11.96 \\
\hline CGCG 049-057 & $30.98 \pm 0.52$ & $7.50_{-0.80}^{+0.84}$ & $11.10_{-0.08}^{+0.07}$ & $31.59 \pm 0.82$ & $2.02_{-0.31}^{+0.34}$ & $3.53_{-0.55}^{+0.59}$ & $10.58_{-0.12}^{+0.11}$ & 10.79 \\
\hline NGC 5936 & $26.55 \pm 0.50$ & $8.95_{-1.09}^{+1.17}$ & $10.73_{-0.10}^{+0.08}$ & $28.43 \pm 0.64$ & $1.44_{-0.21}^{+0.23}$ & $1.97_{-0.29}^{+0.31}$ & $10.14_{-0.12}^{+0.10}$ & 10.48 \\
\hline IRAS F16164-0746 & $31.91 \pm 0.62$ & $12.02_{-1.47}^{+1.56}$ & $11.37_{-0.10}^{+0.08}$ & $32.05 \pm 0.85$ & $3.02_{-0.47}^{+0.51}$ & $5.46_{-0.86}^{+0.99}$ & $10.79_{-013}^{+0.11}$ & 11.01 \\
\hline CGCG 052-037 & $28.57 \pm 0.60$ & $13.65_{-1.86}^{+1.99}$ & $11.12_{-0.11}^{+0.10}$ & $29.95 \pm 0.71$ & $3.57_{-0.53}^{+0.47}$ & $5.54_{-0.83}^{+0.89}$ & $10.67_{-0.12}^{+0.10}$ & 10.97 \\
\hline IRAS F16399-0937 & $29.74 \pm 1.13$ & $12.66_{-3.17}^{+3.80}$ & $11.20_{-0.21}^{+0.11}$ & $29.69 \pm 0.76$ & $4.31_{-0.69}^{+0.75}$ & $6.56_{-1.05}^{+1.14}$ & $10.73_{-0.13}^{+0.11}$ & 11.06 \\
\hline NGC 6285 & $26.13 \pm 0.76$ & $3.19_{-0.57}^{+0.63}$ & $10.24_{-0.15}^{-0.21}$ & $28.41 \pm 0.62$ & $0.83_{-0.12}^{+0.09}$ & $1.14_{-0.16}^{+0.18}$ & $9.89_{-0.12}^{+0.13}$ & 10.17 \\
\hline NGC 6286 & $25.12 \pm 0.31$ & $23.83_{-2.01}^{+2.10}$ & $10.99_{-0.07}^{+0.13}$ & $26.17 \pm 0.53$ & $6.25_{-0.90}^{+0.97}$ & $7.02_{-1.01}^{+1.09}$ & $10.53_{-0.12}^{+0.09}$ & 10.84 \\
\hline IRAS F17138-1017 & $31.49 \pm 0.60$ & $7.82_{-0.93}^{+0.98}$ & $11.16_{-0.09}^{+0.08}$ & $31.33 \pm 0.81$ & $2.46_{-0.38}^{+0.41}$ & $4.22_{-0.65}^{+0.71}$ & $10.64_{-0.12}^{+0.11}$ & 10.94 \\
\hline
\end{tabular}

Notes. While $L_{\mathrm{FIR}}$ is measured between 42.5 and $122.5 \mu \mathrm{m}, L_{\mathrm{TIR}}$ is measured between 3 and $1100 \mu \mathrm{m}$ and is obtained from Eq. (5). We have assumed an uncertainty in $L_{\mathrm{TIR}}$ of $20 \%$. We note that dust masses $M_{\text {dust }}^{\text {fit }}$ are derived evaluating the modified blackbody function using the dust temperature from the models, in contrast with $M_{\text {dust }}^{25 \mathrm{~K}}$, obtained assuming $T_{\text {dust }}=25 \mathrm{~K}$, as suggested in Scoville et al. (2016). The latter are used as the preferred method in this study. 
Table 4. continued.

\begin{tabular}{|c|c|c|c|c|c|c|c|c|}
\hline \multirow[b]{2}{*}{ Source name } & \multicolumn{3}{|c|}{ Overall SED fit } & \multicolumn{5}{|c|}{ Within IRAM-30 $\mathrm{m}$ beam } \\
\hline & $\begin{array}{l}T_{\text {dust }} \\
(\mathrm{K}) \\
\end{array}$ & $\begin{array}{c}M_{\text {dust }}^{\text {fit }} \\
\left(10^{7} M_{\odot}\right)\end{array}$ & $\begin{array}{c}\log \left(L_{\mathrm{FIR}}\right) \\
\left(L_{\odot}\right)\end{array}$ & $\begin{array}{c}T_{\text {dust }} \\
(\mathrm{K}) \\
\end{array}$ & $\begin{array}{c}M_{\mathrm{dust}}^{\mathrm{fit}} \\
\left(10^{7} M_{\odot}\right)\end{array}$ & $\begin{array}{c}M_{\text {dust }}^{25 \mathrm{~K}} \\
\left(10^{7} M_{\odot}\right)\end{array}$ & $\begin{array}{c}\log \left(L_{\mathrm{FIR}}\right) \\
\left(L_{\odot}\right)\end{array}$ & $\begin{array}{c}\log \left(L_{\mathrm{TIR}}\right) \\
\left(L_{\odot}\right)\end{array}$ \\
\hline UGC 11041 & $26.01 \pm 0.47$ & $9.67_{-1.11}^{+1.18}$ & $10.70_{-0.10}^{+0.08}$ & $27.56 \pm 0.60$ & $2.30_{-0.34}^{+0.36}$ & $2.94_{-0.43}^{+0.46}$ & $10.25_{-0.12}^{+0.10}$ & 10.57 \\
\hline CGCG 141-034 & $30.20 \pm 0.57$ & $5.69_{-0.70}^{+0.74}$ & $10.90_{-0.10}^{+0.08}$ & $30.04 \pm 0.73$ & $2.01_{-0.30}^{+0.33}$ & $3.14_{-0.47}^{+0.51}$ & $10.43_{-0.12}^{+0.10}$ & 10.70 \\
\hline IRAS $18090+0130$ & $28.06 \pm 0.92$ & $4.78_{-1.08}^{+1.21}$ & $10.61_{-0.19}^{+0.15}$ & $27.49 \pm 0.62$ & $7.39_{-1.12}^{+1.22}$ & $9.39_{-1.43}^{+1.54}$ & $10.74_{-0.13}^{+0.10}$ & 11.06 \\
\hline NGC 6701 & $27.13 \pm 0.46$ & $8.04_{-0.88}^{+0.93}$ & $10.75_{-0.09}^{+0.07}$ & $28.87 \pm 0.67$ & $1.50_{-0.22}^{+0.24}$ & $2.13_{-0.32}^{+0.34}$ & $10.20_{-0.12}^{+0.10}$ & 10.52 \\
\hline NGC 6786 & $27.52 \pm 0.47$ & $9.49_{-1.07}^{+1.14}$ & $10.86_{-0.10}^{+0.07}$ & $28.52 \pm 0.64$ & $2.78_{-0.41}^{+0.42}$ & $3.86_{-0.56}^{+0.61}$ & $10.43_{-0.12}^{+0.10}$ & 10.74 \\
\hline UGC 11415 & $29.03 \pm 0.58$ & $4.76_{-0.59}^{+0.03}$ & $10.71_{-0.10}^{+0.10}$ & $30.18 \pm 0.73$ & $1.59_{-0.24}^{-0.41}$ & $2.51_{-0.38}^{+0.50}$ & $10.34_{-0.12}^{+0.12}$ & 10.72 \\
\hline ESO 593-IG008 & $28.57 \pm 0.54$ & $46.04_{-5.57}^{+5.93}$ & $11.63_{-0.11}^{+0.07}$ & $28.74 \pm 0.67$ & $16.27_{-2.46}^{+2.66}$ & $23.10_{-3.50}^{+3.70}$ & $11.20_{-0.13}^{+0.09}$ & 11.50 \\
\hline NGC 6907 & $24.47 \pm 0.51$ & $14.87_{-1.94}^{+2.09}$ & $10.71_{-0.11}^{+0.09}$ & $26.75 \pm 0.57$ & $1.24_{-0.18}^{+0.20}$ & $1.47_{-0.22}^{+0.33}$ & $9.90_{-0.12}^{+0.10}$ & 10.25 \\
\hline IRAS $21101+5810$ & $32.71 \pm 0.77$ & $11.78_{-1.79}^{+1.92}$ & $11.42_{-0.12}^{+0.11}$ & $28.26 \pm 0.73$ & $6.31_{-1.06}^{+1.15}$ & $8.59_{-1.44}^{+1.57}$ & $10.75_{-0.14}^{+0.12}$ & 11.07 \\
\hline ESO 602-G025 & $27.22 \pm 0.51$ & $13.79_{-1.71}^{+1.82}$ & $10.99_{-0.10}^{+0.12}$ & $28.75 \pm 0.67$ & $3.92_{-0.59}^{+0.06}$ & $5.55_{-0.83}^{+0.90}$ & $10.60_{-0.12}^{+0.14}$ & 10.92 \\
\hline UGC 12150 & $28.18 \pm 0.36$ & $11.61_{-0.97}^{+1.02}$ & $11.01_{-0.07}^{+0.05}$ & $28.87 \pm 0.67$ & $3.99_{-0.60}^{+0.65}$ & $5.69_{-0.85}^{+0.92}$ & $10.62_{-0.12}^{+0.10}$ & 10.93 \\
\hline IRAS F22491-1808 & $37.05 \pm 0.83$ & $17.27_{-2.10}^{+2.23}$ & $11.88_{-0.10}^{+0.09}$ & $36.99 \pm 1.15$ & $7.91_{-1.31}^{+1.42}$ & $19.93_{-3.29}^{+3.58}$ & $11.54_{-0.13}^{+0.12}$ & 11.75 \\
\hline CGCG 453-062 & $28.28 \pm 0.62$ & $12.32_{-1.80}^{+1.94}$ & $11.05_{-0.12}^{+0.09}$ & $29.26 \pm 0.70$ & $3.87_{-0.59}^{+0.64}$ & $5.71_{-0.87}^{+0.94}$ & $10.64_{-0.12}^{+0.10}$ & 10.92 \\
\hline 2MASX J23181352+0633267 & $25.38 \pm 0.77$ & $11.07_{-2.08}^{+2.30}$ & $10.69_{-0.16}^{+0.13}$ & $23.42 \pm 0.40$ & $0.38_{-0.05}^{+0.05}$ & $0.32_{-0.04}^{+0.05}$ & $8.98_{-0.11}^{+0.08}$ & 9.46 \\
\hline
\end{tabular}

as found by Gao \& Solomon (2004), where a clear super-linear slope is derived.

A way of interpreting the relation between infrared and $\mathrm{CO}$ luminosities is through the star formation efficiency, which is the ratio of the total energy from young massive stars per unit of star-forming molecular gas, and which is usually represented by $L_{\mathrm{TIR}, \mathrm{FIR}} / L_{\mathrm{CO}}^{\prime}$. However, the use of $L_{\mathrm{CO}}^{\prime}$ can be misleading, as it cannot be directly converted into a $M_{\mathrm{H}_{2}}$ estimate without the use of an $\alpha$ conversion factor, which we showed to vary between the samples in Sect. 5.1.

We can rework the above in terms of the gas depletion timescales, $t_{\text {dep }}=M\left(\mathrm{H}_{2}\right) / \mathrm{SFR}$, where the molecular gas mass is derived using $\alpha=1.8 M_{\odot}\left(\mathrm{K} \mathrm{km} \mathrm{s}^{-1} \mathrm{pc}^{2}\right)^{-1}$ for (U)LIRGs (see Sect. 5.1), and the Milky Way value for the local sample. The star formation rate (SFR) is obtained from the infrared and UV luminosity (see Murphy et al. 2011)

$$
\frac{\mathrm{SFR}}{M_{\odot} \mathrm{yr}^{-1}}=4.42 \times 10^{-44}\left(\frac{L_{\mathrm{FUV}}+0.88 L_{\mathrm{FIR}}}{\mathrm{erg} \mathrm{s}^{-1}}\right),
$$

where we have used our aperture-matched, SED-derived FIR luminosity, while the UV luminosities (integrated luminosity within the GALEX far ultraviolet (FUV) filter; $\lambda_{\mathrm{eff}}=1528 \AA$ ) were obtained from Howell et al. (2010) for the (U)LIRGs, and from the GALEX Ultraviolet Atlas of Nearby Galaxies (Gil de Paz et al. 2007) for the local comparison sample. Most of the UV emission is expected to be inside the IRAM-30 $\mathrm{m}$ beam, thus any correction would be within the errors.

Figure 6 presents the variation of $t_{\text {dep }}$ as a function of $L_{\mathrm{TIR}, \mathrm{FIR}}$. Fits to the trends considering all three samples combined yield the correlations

$\log \left(t_{\mathrm{dep}}\right)=(-0.19 \pm 0.07) \log \left(L_{\mathrm{TIR}}\right)+(10.89 \pm 0.77)$

$\log \left(t_{\mathrm{dep}}\right)=(-0.20 \pm 0.06) \log \left(L_{\mathrm{FIR}}\right)+(10.95 \pm 0.67)$,

with median Pearson correlation coefficients of $\rho=-0.396$ and $\rho=-0.454$, respectively, and $p$-values of 0.006 and 0.001 , showing a moderately strong statistical significance.
We obtain a median $t_{\text {dep }}$ of 1.3 Gyr for the local sample, compared to the 2.1 Gyr from Kennicutt (1998) or 2.35 Gyr from Bigiel et al. (2011). This timescale is obtained assuming there is not gas replenishing and that there is a constant star formation rate. We note that $t_{\text {dep }}$ increases by a factor of two to three if interstellar gas recycling is considered (Ostriker \& Thuan 1975; Kennicutt et al. 1994). Nevertheless, the above correlation shows that $L_{\mathrm{TIR}}=10^{11} L_{\odot}$ LIRGs would deplete their available gas in $\sim 630 \mathrm{Myr}$, whereas $L_{\mathrm{TIR}}=10^{12} L_{\odot}$ ULIRGs would deplete their gas in $\sim 400 \mathrm{Myr}$, a period more than three times shorter than for local normal spiral galaxies. The compression of the molecular gas clouds due to large-scale shocks, which would enhance star formation, has been proposed to account for this difference (Jog \& Solomon 1992; Barnes 2004; Genzel et al. 2010). However, the quoted depletion times are obtained from the total molecular gas (as traced by ${ }^{12} \mathrm{CO}$ ), and not specifically from the dense gas phase of the molecular medium, which is expected to be more closely associated to regions where star formation is imminent.

We note the strong dependence of depletion time (Eq. (9)) with $\alpha_{\mathrm{CO}}$. Indeed, if dust masses were derived using the fitted $T_{\text {dust }}$ instead of the fixed $25 \mathrm{~K}$, the relation would be $\log \left(t_{\text {dep }}\right)=$ $-0.36 \log \left(L_{\mathrm{TIR}}\right)+12.46$, implying an even larger difference (one order of magnitude) in the depletion time between (U)LIRGs and the comparison local sample.

Finally, if fitted $T_{\text {dust }}$ are used to estimate the dust masses, our data also show a clear correlation between the depletion time and the dust temperature as shown in Fig. 7. The fit of the combined data yields

$\log \left(t_{\text {dep }}\right)=(-6.9 \pm 0.8) \times 10^{-2} T_{\text {dust }}+(10.6 \pm 0.2)$,

with $\rho=-0.812$, and a robust statistical significance ( $p$-value of $1.1 \times 10^{-7}$ ). This trend shows a very sensitive variation, with depleting times being one order of magnitude lower with an increase of $\sim 15 \mathrm{~K}$. In other words, the star formation efficiency $\left(\propto 1 / t_{\mathrm{dep}}\right)$ increases by a factor of ten by such variation in the dust temperature. 


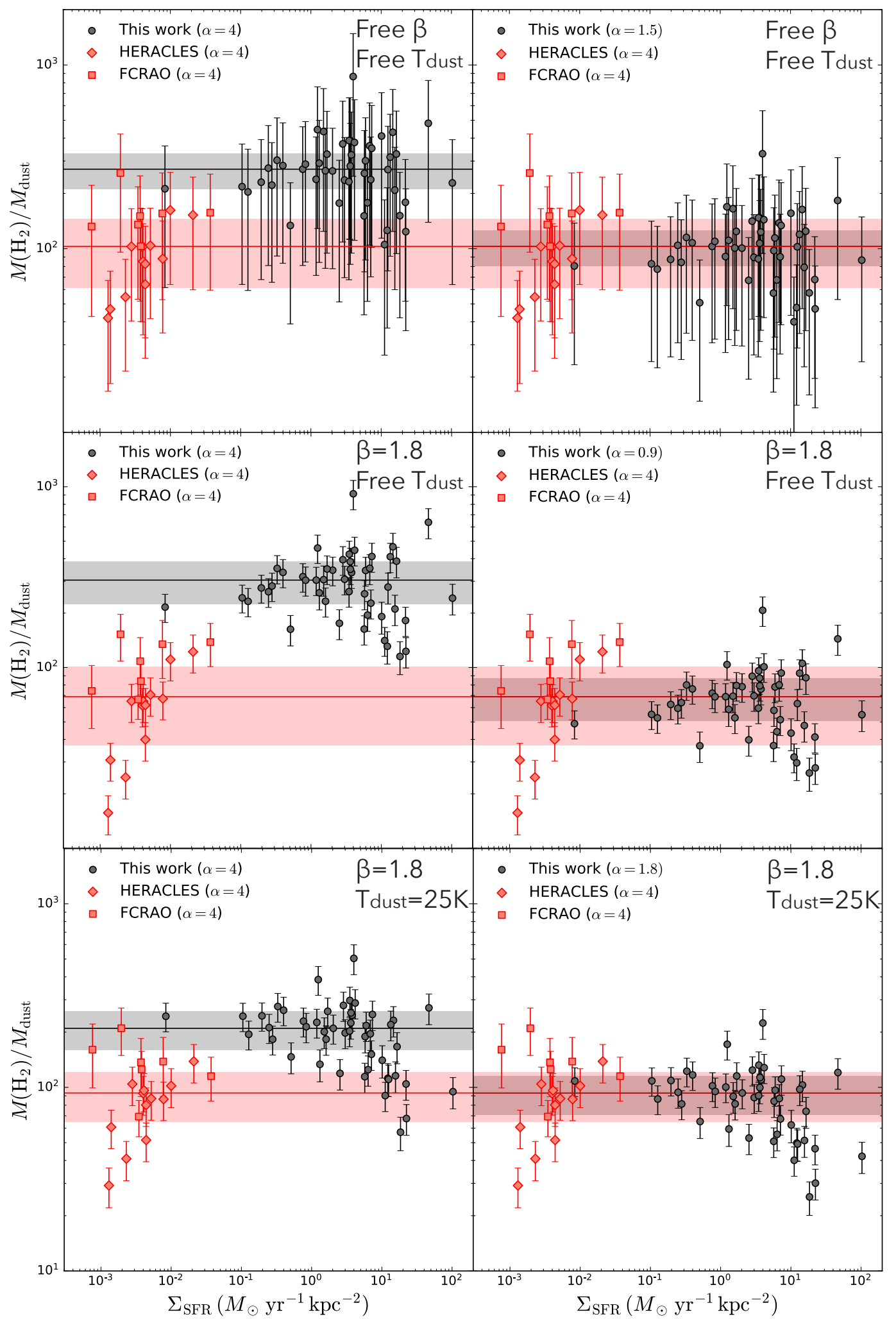

Fig. 4. Gas-to-dust mass ratio for both the reference sample, consisting of HERACLES (red diamonds) and FCRAO (red squares) sources, and our (U)LIRG sample (black circles) as a function of $\Sigma_{\text {SFR. }}$. Horizontal lines show the median value for each sample, and shaded areas indicate their median absolute deviations. Top panels: $M_{\text {dust }}$ has been obtained leaving $\beta$ as a free parameter in the SED fit and using the fitted $T_{\text {dust }}$ to derive dust mass. Middle panels: emissivity index has been fixed to $\beta=1.8$, but $M_{\text {dust }}$ is still obtained from the fitted dust temperatures. Finally, in the bottom panels, emissivity is fixed to 1.8 , and dust masses are obtained assuming a fixed $25 \mathrm{~K}$ dust temperature (see main text for further details). Left panels: results of using a Milky Way CO-to- $\mathrm{H}_{2}$ conversion factor $(\alpha=4)$. To make the gas-to-dust mass ratio of (U)LIRGs consistent with the reference sample, $\alpha$ has been modified to the value quoted in the right panels. For the remainder of this work, a fixed dust emissivity of $\beta=1.8$ is assumed, and we adopt a CO-to- $\mathrm{H}_{2}$ factor of $\alpha=1.8$ for (U)LIRGs, as required to make the median gas-to-dust mass ratio of (U)LIRGs match that of the reference sample of (non-LIRG) spiral galaxies. 


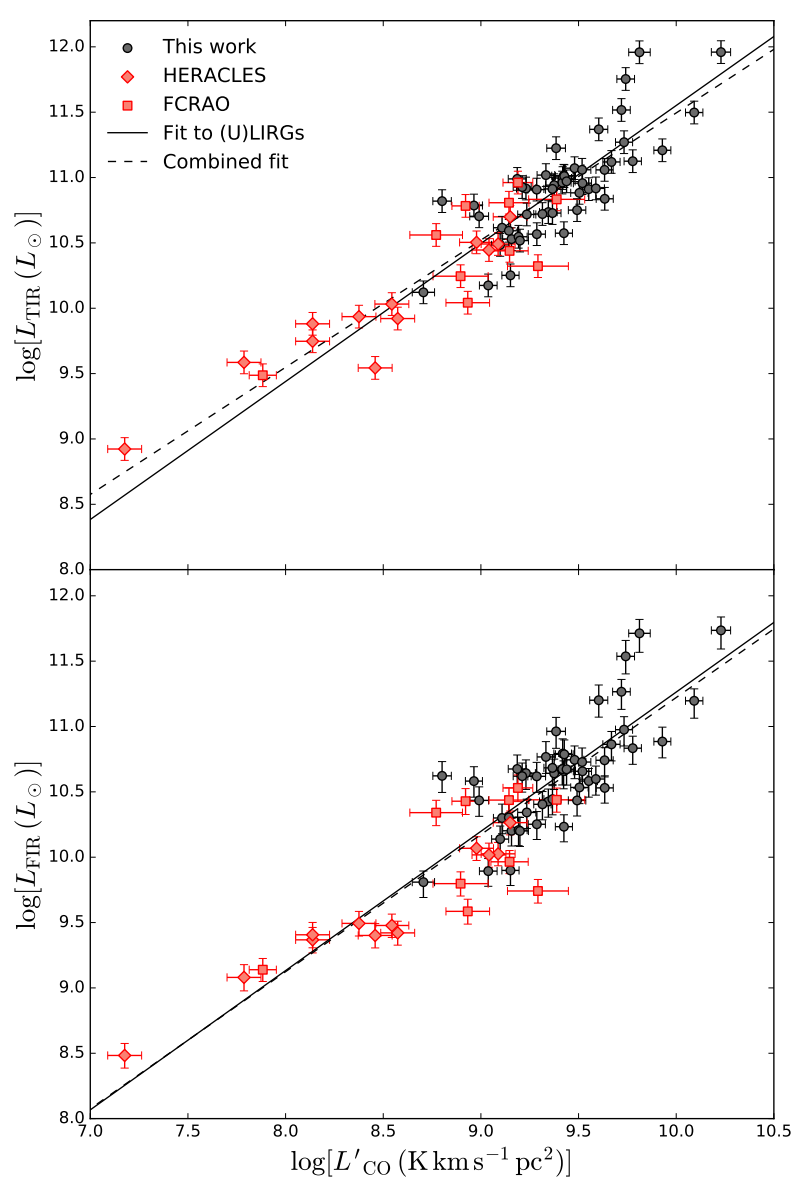

Fig. 5. Total and far IR luminosities versus the CO luminosity. Solid lines show linear fits to our (U)LIRG sample, yielding $\log \left(L_{\mathrm{TIR}}\right)=$ $(1.05 \pm 0.12) \log \left(L_{\mathrm{CO}}^{\prime}\right)+(1.02 \pm 1.12)$ and $\log \left(L_{\mathrm{FIR}}\right)=(1.07 \pm$ $0.13) \log \left(L_{\mathrm{CO}}^{\prime}\right)+(0.55 \pm 1.26)$. Dashed lines are fits including the comparison samples, which also show linear slopes when using $L_{\mathrm{TIR}}$ $(0.97 \pm 0.06)$ or $L_{\mathrm{FIR}}(1.05 \pm 0.06)$. To avoid AGN contamination, only those (U)LIRGs with an AGN bolometric fraction $<0.20$ (obtained from Díaz-Santos et al. 2017) were plotted and fitted.

\subsection{The ${ }^{12} \mathrm{CO} /{ }^{13} \mathrm{CO}$ line intensity ratio}

In this sub-section, we examine the relationship between the ${ }^{12} \mathrm{CO} /{ }^{13} \mathrm{CO}$ line intensity ratio and the dust temperature. In Fig. 8 we present a plot of ${ }^{12} \mathrm{CO} /{ }^{13} \mathrm{CO}$ for the (U)LIRGs in our sample versus the $60 \mu \mathrm{m}-100 \mu \mathrm{m}$ IRAS flux density ratio $f_{60} / f_{100}$, which is used as a proxy for the dust temperature. We do not use here our SED-fit-derived $T_{\text {dust }}$ so as to be able to systematically compare with previous studies on LIRGs (for which Herschel photometry is not available). We have confirmed nonetheless the very good correlation between $f_{60} / f_{100}$ and the derived $T_{\text {dust }}$ for our sample, finding $T_{\text {dust }}=(14.1 \pm 1.2)$ $\left(f_{60} / f_{100}\right)+(20.6 \pm 0.8)$. The data from the present study are compared with published results from Aalto et al. (1995) and Costagliola et al. (2011). The former is formed of 31 local spiral galaxies, starbursts, interacting systems, and luminous mergers, while the latter includes a representation of 12 Seyferts, starbursts, and LIRGs. Our results are in agreement with those studies, finding lower values of ${ }^{12} \mathrm{CO} /{ }^{13} \mathrm{CO}$ for $f_{60} / f_{100}<0.8$, and a relatively steep enhancement for $f_{60} / f_{100}>0$.8. This cutoff point corresponds to $\sim 32 \mathrm{~K}$. This general trend is also in agreement with Milky Way measurements of molecular clouds (Barnes et al. 2015). Only two of the sources in our sample present a lowerlimit value that seems at odds with this behavior, showing too large
${ }^{12} \mathrm{CO} /{ }^{13} \mathrm{CO}$ ratios $(>21.4$ and $>27.6)$ for their FIR color $(0.48$ and 0.59). These are NGC 6907 and UGC 05101, and they are marked in Fig. 8 with their names.

The above relation can be explained as a result of the decreasing optical depth of the ${ }^{12} \mathrm{CO}$ line with increasing ISM temperature (e.g., Young \& Sanders 1986; Aalto et al. 1995). Increased linewidths in diffuse or turbulent gas may also result in decreased line optical depths and elevated line ratios (see, e.g., Polk et al. 1988; Aalto et al. 1995, 2010; König et al. 2016).

In addition to optical depth effects, the relative ${ }^{12} \mathrm{CO}$ and ${ }^{13} \mathrm{CO}$ abundances ratio will impact the intensity ratio. The less abundant isotopomer of ${ }^{13} \mathrm{CO}$ will become selectively photodissociated in diffuse gas since it cannot self-shield like ${ }^{12} \mathrm{CO}$. Furthermore, stellar nucleosynthesis will alter the ${ }^{12} \mathrm{C} /{ }^{13} \mathrm{C}$ abundance ratio and low-metallicity gas is expected to have high ${ }^{12} \mathrm{C} /{ }^{13} \mathrm{C}$ ratios (see, e.g., Casoli et al. 1992; Henkel et al. 2014; Tang et al. 2019). The ejecta of massive stars are also expected to have higher ${ }^{12} \mathrm{C} /{ }^{13} \mathrm{C}$ abundance ratios, which means that in the early stages of a starburst ${ }^{12} \mathrm{CO} /{ }^{13} \mathrm{CO}$ abundance ratios may be higher. Indeed, there seems to be an enrichment of the ISM due to a young starburst and/or a top-heavy initial mass function (Sliwa et al. 2017).

\section{Summary}

We observed 55 (U)LIRGs with the IRAM-30 m Telescope. In this work, we have focused on the ${ }^{12} \mathrm{CO}(1-0)$ transition as a tracer of molecular gas. We summarize our results as follows:

1. We observed ${ }^{12} \mathrm{CO}$ and ${ }^{13} \mathrm{CO}$ simultaneously, in the same band, obtaining detection rates of $96 \%$ and $56 \%$ for ${ }^{12} \mathrm{CO}$ and ${ }^{13} \mathrm{CO}$, respectively, as expected from ${ }^{13} \mathrm{CO}$ being much less abundant than ${ }^{12} \mathrm{CO}$.

2. We have used Herschel data to construct the far infrared SED of the emitting region inside the IRAM- $30 \mathrm{~m}$ beam for each galaxy in our sample, and fitted the SED to a modified blackbody model, obtaining the dust masses and temperatures, as well as $L_{\text {FIR }}$. We also have systematically fitted local galaxies from HERACLES and FCRAO, for which both CO and Herschel data are available, to be used as a local comparison sample. Complementarily, we have used MIPS data to derive the total IR luminosities, $L_{\mathrm{TIR}}$, for both samples.

3. We have determined an average $\mathrm{CO}-$ to- $\mathrm{H}_{2}$ conversion factor for (U)LIRGs of $\langle\alpha\rangle=1.8_{-0.8}^{+1.3} M_{\odot}\left(\mathrm{K} \mathrm{km} \mathrm{s}^{-1} \mathrm{pc}^{2}\right)^{-1}$, $\sim 2.2$ times smaller than the accepted conversion factor for local spiral galaxies, for an assumed constant gas-to-dust mass ratio between the local comparison sample and the (U)LIRGs sample. This value is obtained by assuming a fixed dust temperature of $25 \mathrm{~K}$ for every source. If the SED fitted temperatures are used instead, the average value of $\alpha$ for (U)LIRGs would be $0.9_{-0.5}^{+0.9}$.

4. We confirm the close linear correlation between the $\mathrm{CO}$ luminosity and $L_{\text {TIR,FIR }}$. We have also found an enhancement in the star formation efficiency for more IR-luminous systems. Consequently, the depletion time for these systems is shorter, following a $\log -\log$ correlation with $L_{\mathrm{IR}, \mathrm{TIR}}$. However, this effect with $t_{\text {dep }}$ is not observed in the higher density phase of molecular gas. When $M_{\text {dust }}$ are derived from fitted $T_{\text {dust }}$, the $t_{\text {dep }}$ also shows a clear decreasing trend with dust temperature, implying an increase of one order of magnitude in the star formation efficiency per increase of $\sim 15 \mathrm{~K}$.

5 . We have re-examined the variation between the ratio of ${ }^{12} \mathrm{CO}$ and its isotopologue ${ }^{13} \mathrm{CO}$ with the dust temperature, as traced by the proxy $f_{60} / f_{100}$. Our data confirm previous results, with cooler galaxies having lower ${ }^{12} \mathrm{CO} /{ }^{13} \mathrm{CO}$ ratios, with a steep increase above $f_{60} / f_{100} \sim 0.8$. 


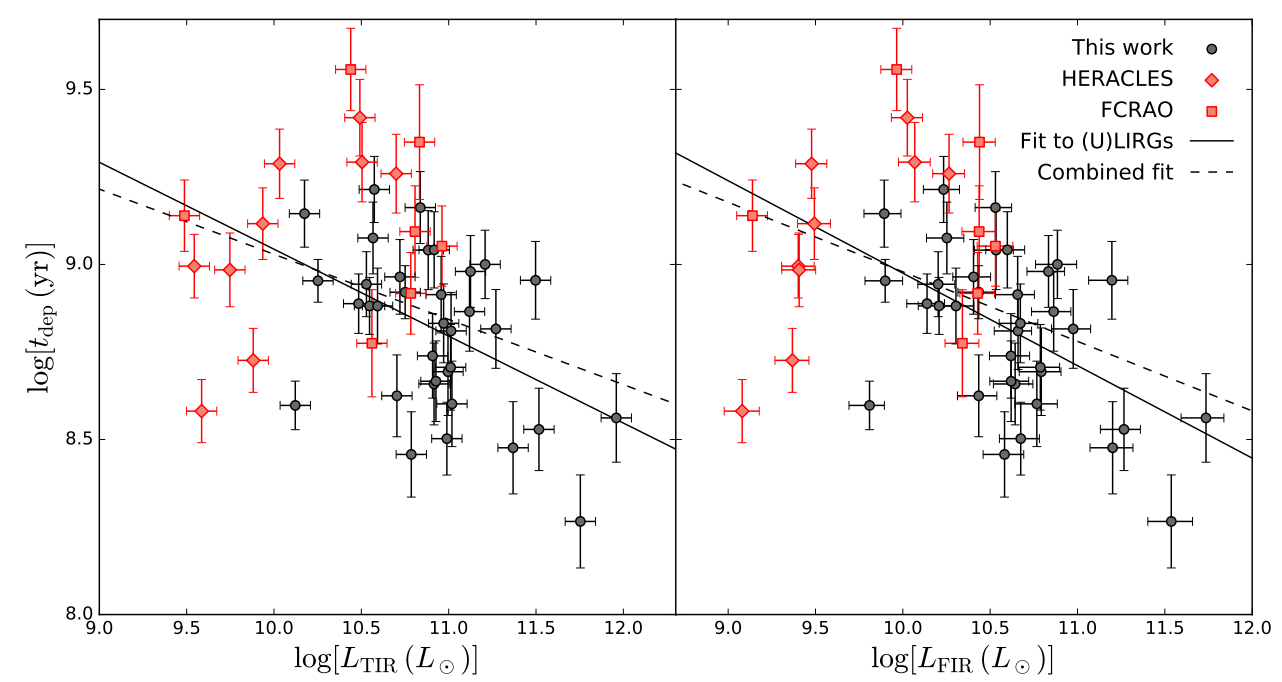

Fig. 6. Depletion time as a function of TIR and FIR luminosities. Solid lines are fits to the (U)LIRG sample, where $\log \left(t_{\mathrm{dep}}\right)=(-0.25 \pm 0.10) \log \left(L_{\mathrm{TIR}}\right)+$ $(11.54 \pm 1.05)$ and $\log \left(t_{\text {dep }}\right)=(-0.26 \pm$ $0.09) \log \left(L_{\mathrm{FIR}}\right)+(11.61 \pm 0.96)$. Dashed lines are a fit to the combined sample and yield $\log \left(t_{\mathrm{dep}}\right)=(-0.19 \pm 0.07) \log \left(L_{\mathrm{TIR}}\right)+$ $(10.89 \pm 0.77)$ and $\log \left(t_{\text {dep }}\right)=(-0.20 \pm$ $0.06) \log \left(L_{\mathrm{FIR}}\right)+(10.95 \pm 0.67)$. This trend implies that gas is consumed faster in (U)LIRGs and thus star formation is more efficient in these systems, compared to lower luminosity spiral galaxies.

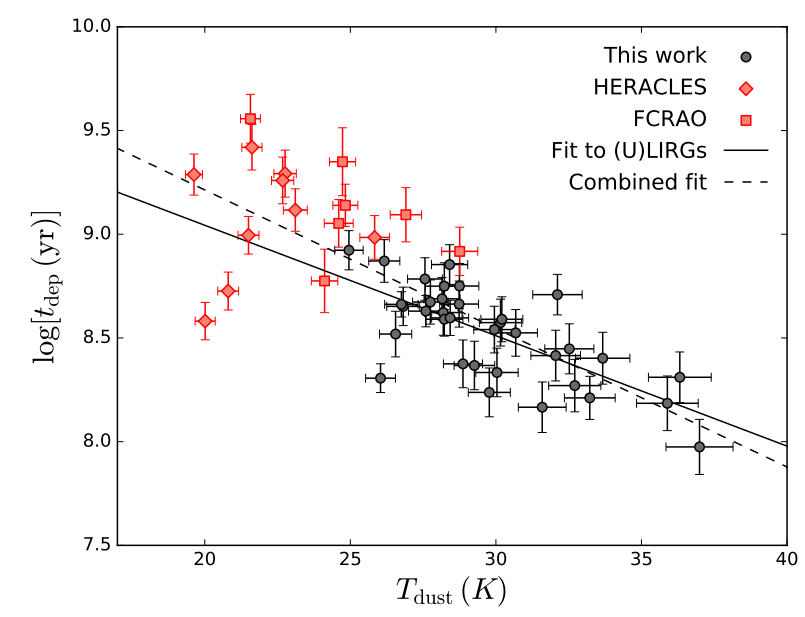

Fig. 7. Depletion time versus dust temperature, using $T_{\text {dust }}$ fitted values to obtain $M_{\text {dust }}$. Dust tmeperature is obtained from the SED model fit. The solid line represents the linear fit for the (U)LIRGs, yielding $\log \left(t_{\text {dep }}\right)=(-5.5 \pm 1.0) \times 10^{-2} T_{\text {dust }}+(10.1 \pm 0.3)$. The dash lines represent the best fit considering also our local comparison sample; the fit yields $\log \left(t_{\text {dep }}\right)=(-6.9 \pm 0.8) \times 10^{-2} T_{\text {dust }}+(10.6 \pm 0.2)$,

Acknowledgements. We thank the anonymous referee for the useful comments. We also thank Nick Scoville for his discussion and comments on the paper. We are grateful to Manuel González for his help in the preparation of the scripts and during the observations. This work is based on observations carried out under project numbers 099-10, 092-11, 227-11, 076-12, 222-13, and D01-13 with the $30 \mathrm{~m}$ telescope. IRAM is supported by INSU/CNRS (France), MPG (Germany), and IGN (Spain). We thank the director of IRAM 30m for the approval of the discretionary time requested. RHI, MAPT, and AA acknowledge support from the Spanish MINECO through grants AYA2012-38491-C0202 and AYA2015-63939-C2-1-P. G.C.P. was supported by a FONDECYT Postdoctoral Fellowship (No. 3150361). G.C.P. also acknowledges support from the University of Florida. G.C.P. and A.S.E. were supported by the NSF grant AST 1109475. A.S.E. was also supported by the Taiwan, R.O.C. Ministry of Science and technology grant MoST 102-2119-M-001-MY3. T.D.-S. acknowledges support from ALMA-CONICYT project 31130005 and FONDECYT regular project 1151239. This work was supported in part by National Science Foundation grant No. PHYS-1066293 and the hospitality of the Aspen Center for Physics. This research has made use of the NASA/IPAC Extragalactic Database (NED), which is operated by the Jet Propulsion Laboratory, California Institute of Technology, under contract with the National Aeronautics and Space Administration.

\section{References}

Aalto, S., Booth, R. S., Black, J. H., \& Johansson, L. E. B. 1995, A\&A, 300, 369 Aalto, S., Beswick, R., \& Jütte, E. 2010, A\&A, 522, A59

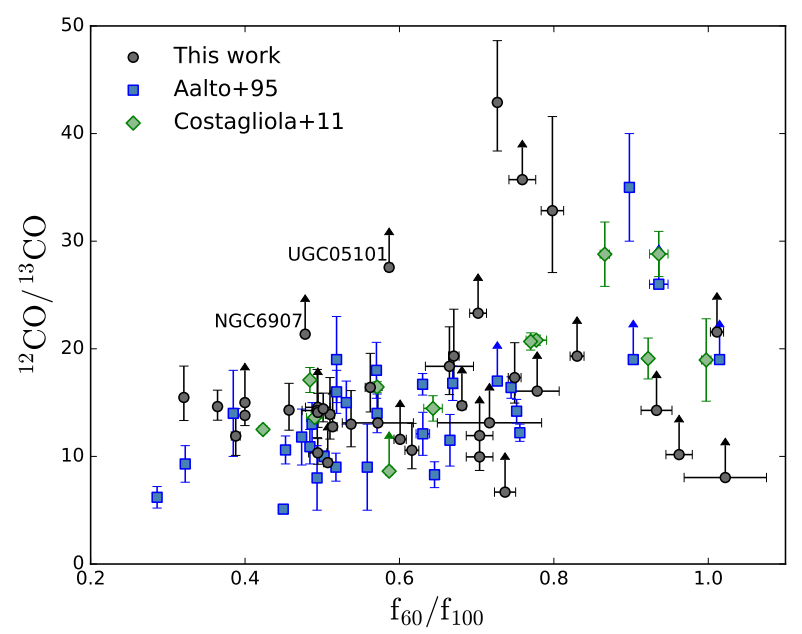

Fig. 8. ${ }^{12} \mathrm{CO}$ over ${ }^{13} \mathrm{CO}$ intensity ratio plotted vs. the IRAS flux ratio $f_{60} / f_{100}$, used as a proxy for the dust temperature. For the line ratios, the uncertainties were derived using a Monte Carlo simulation using 10000 samples and following a similar prescription to Crocker et al. (2012). The sources are from different samples: this work (black circles), Aalto et al. (1995, blue squares), and Costagliola et al. (2011, green diamonds). Errors are plotted at $1 \sigma$ level, although $\mathrm{x}$-axis errors are not appreciable for many sources. The two labeled sources are at odds with the rest of the sample for their observed $f_{60} / f_{100}$ ratio.

Armus, L., Mazzarella, J. M., Evans, A. S., et al. 2009, PASP, 121, 559 Barnes, J. E. 2004, MNRAS, 350, 798

Barnes, P. J., Muller, E., Indermuehle, B., et al. 2015, ApJ, 812, 6 Bianchi, S. 2013, A\&A, 552, A89

Bigiel, F., Leroy, A. K., Walter, F., et al. 2011, ApJ, 730, L13

Blain, A. W., Barnard, V. E., \& Chapman, S. C. 2003, MNRAS, 338,733 Bolatto, A. D., Wolfire, M., \& Leroy, A. K. 2013, ARA\&A, 51, 207 Bryant, P. M., \& Scoville, N. Z. 1999, AJ, 117, 2632

Carter, M., Lazareff, B., Maier, D., et al. 2012, A\&A, 538, A89

Casoli, F., Dupraz, C., \& Combes, F. 1992, A\&A, 264, 55

Chu, J. K., Sanders, D. B., Larson, K. L., et al. 2017, ApJS, 229, 25 Cortese, L., Bekki, K., Boselli, A., et al. 2016, MNRAS, 459, 3574 Costagliola, F., Aalto, S., Rodriguez, M. I., et al. 2011, A\&A, 528, A30 Crocker, A., Krips, M., Bureau, M., et al. 2012, MNRAS, 421, 1298 Daddi, E., Elbaz, D., Walter, F., et al. 2010, ApJ, 714, L118

Dale, D. A., \& Helou, G. 2002, ApJ, 576, 159

Dale, D. A., Gil de Paz, A., Gordon, K. D., et al. 2007, ApJ, 655, 863 Dale, D. A., Cohen, S. A., Johnson, L. C., et al. 2009, ApJ, 703, 517 Dale, D. A., Aniano, G., Engelbracht, C. W., et al. 2012, ApJ, 745, 95 Davies, J. I., Bianchi, S., Cortese, L., et al. 2012, MNRAS, 419, 3505 Díaz-Santos, T., Alonso-Herrero, A., Colina, L., et al. 2010, ApJ, 711, 328 
Díaz-Santos, T., Charmandaris, V., Armus, L., et al. 2011, ApJ, 741, 32 Díaz-Santos, T., Armus, L., Charmandaris, V., et al. 2017, ApJ, 846, 32 Downes, D., \& Solomon, P. M. 1998, ApJ, 507, 615

Draine, B. T., \& Lee, H. M. 1984, ApJ, 285, 89

Engelbracht, C. W., Rieke, G. H., Gordon, K. D., et al. 2008, ApJ, 678, 804 Gao, Y., \& Solomon, P. M. 2004, ApJ, 606, 271

Genzel, R., Tacconi, L. J., Gracia-Carpio, J., et al. 2010, MNRAS, 407, 2091

Gil de Paz, A., Boissier, S., Madore, B. F., et al. 2007, ApJS, 173, 185

Greve, T. R., Leonidaki, I., Xilouris, E. M., et al. 2014, ApJ, 794, 142

Haan, S., Surace, J. A., Armus, L., et al. 2011, AJ, 141, 100

Henkel, C., Asiri, H., Ao, Y., et al. 2014, A\&A, 565, A3

Hinshaw, G., Larson, D., Komatsu, E., et al. 2013, ApJS, 208, 19

Howell, J. H., Armus, L., Mazzarella, J. M., et al. 2010, ApJ, 715, 572

Iwasawa, K., Sanders, D. B., Teng, S. H., et al. 2011, A\&A, 529, A106

Jog, C. J., \& Solomon, P. M. 1992, ApJ, 387, 152

Kelly, B. C. 2007, ApJ, 665, 1489

Kennicutt, Jr., R. C. 1998, ARA\&A, 36, 189

Kennicutt, Jr., R. C., Tamblyn, P., \& Congdon, C. E. 1994, ApJ, 435, 22

Kim, D.-C., Evans, A. S., Vavilkin, T., et al. 2013, ApJ, 768, 102

König, S., Aalto, S., Muller, S., et al. 2016, A\&A, 594, A70

Leroy, A. K., Walter, F., Brinks, E., et al. 2008, AJ, 136, 2782

Leroy, A. K., Walter, F., Bigiel, F., et al. 2009, AJ, 137, 4670

Leroy, A. K., Walter, F., Sandstrom, K., et al. 2013, AJ, 146, 19

Murphy, E. J., Condon, J. J., Schinnerer, E., et al. 2011, ApJ, 737, 67

Narayanan, D., Groppi, C. E., Kulesa, C. A., \& Walker, C. K. 2005, ApJ, 630, 269

Ostriker, J. P., \& Thuan, T. X. 1975, ApJ, 202, 353

Papadopoulos, P. P., van der Werf, P. P., Xilouris, E. M., et al. 2012a, MNRAS, 426, 2601

Papadopoulos, P. P., van der Werf, P., Xilouris, E., Isaak, K. G., \& Gao, Y. 2012b, ApJ, 751, 10
Petric, A. O., Armus, L., Howell, J., et al. 2011, ApJ, 730, 28 Planck Collaboration XXI. 2011, A\&A, 536, A21

Polk, K. S., Knapp, G. R., Stark, A. A., \& Wilson, R. W. 1988, ApJ, 332, 432 Privon, G. C., Herrero-Illana, R., Evans, A. S., et al. 2015, ApJ, 814, 39

Rémy-Ruyer, A., Madden, S. C., Galliano, F., et al. 2014, A\&A, 563, A31

Sanders, D. B., Scoville, N. Z., \& Soifer, B. T. 1991, ApJ, 370, 158

Sanders, D. B., Mazzarella, J. M., Kim, D.-C., Surace, J. A., \& Soifer, B. T. 2003 , AJ, 126, 1607

Scoville, N. Z., \& Sanders, D. B. 1987, in Interstellar Processes, eds. D. J. Hollenbach, \& H. A. Thronson, Jr. , Astrophys. Space Sci. Libr., 134, 21

Scoville, N., Sheth, K., Walter, F., et al. 2015, ApJ, 800, 70

Scoville, N., Sheth, K., Aussel, H., et al. 2016, ApJ, 820, 83

Sliwa, K., Wilson, C. D., Aalto, S., \& Privon, G. C. 2017, ApJ, 840, L11

Soifer, B. T., Sanders, D. B., Neugebauer, G., et al. 1986, ApJ, 303, L41

Solomon, P. M., Downes, D., \& Radford, S. J. E. 1992, ApJ, 398, L29

Solomon, P. M., Downes, D., Radford, S. J. E., \& Barrett, J. W. 1997, ApJ, 478, 144

Stierwalt, S., Armus, L., Surace, J. A., et al. 2013, ApJS, 206, 1

Stierwalt, S., Armus, L., Charmandaris, V., et al. 2014, ApJ, 790, 124

Tang, X. D., Henkel, C., Menten, K. M., et al. 2019, A\&A, in press, https: //doi.org/10.1051/0004-6361/201935603

Tinney, C. G., Scoville, N. Z., Sanders, D. B., \& Soifer, B. T. 1990, ApJ, 362, 473

Torrey, P., Vogelsberger, M., Hernquist, L., et al. 2018, MNRAS, 477, L16

Tremonti, C. A., Heckman, T. M., Kauffmann, G., et al. 2004, ApJ, 613, 898

Wilson, C. D., Petitpas, G. R., Iono, D., et al. 2008, ApJS, 178, 189

Yamashita, T., Komugi, S., Matsuhara, H., et al. 2017, ApJ, 544, 96

Yao, L., Seaquist, E. R., Kuno, N., \& Dunne, L. 2003, ApJ, 588, 771

Young, J. S., \& Sanders, D. B. 1986, ApJ, 302, 680

Young, J. S., Xie, S., Tacconi, L., et al. 1995, ApJS, 98, 219 


\section{Appendix A: Observed spectra and fitted Gaussian line components}

Figure A.1 presents the same results as Fig. 2 for our complete sample. We have also tabulated in Table A.1 the Gaussian components that were fitted to the ${ }^{12} \mathrm{CO}$ spectra.
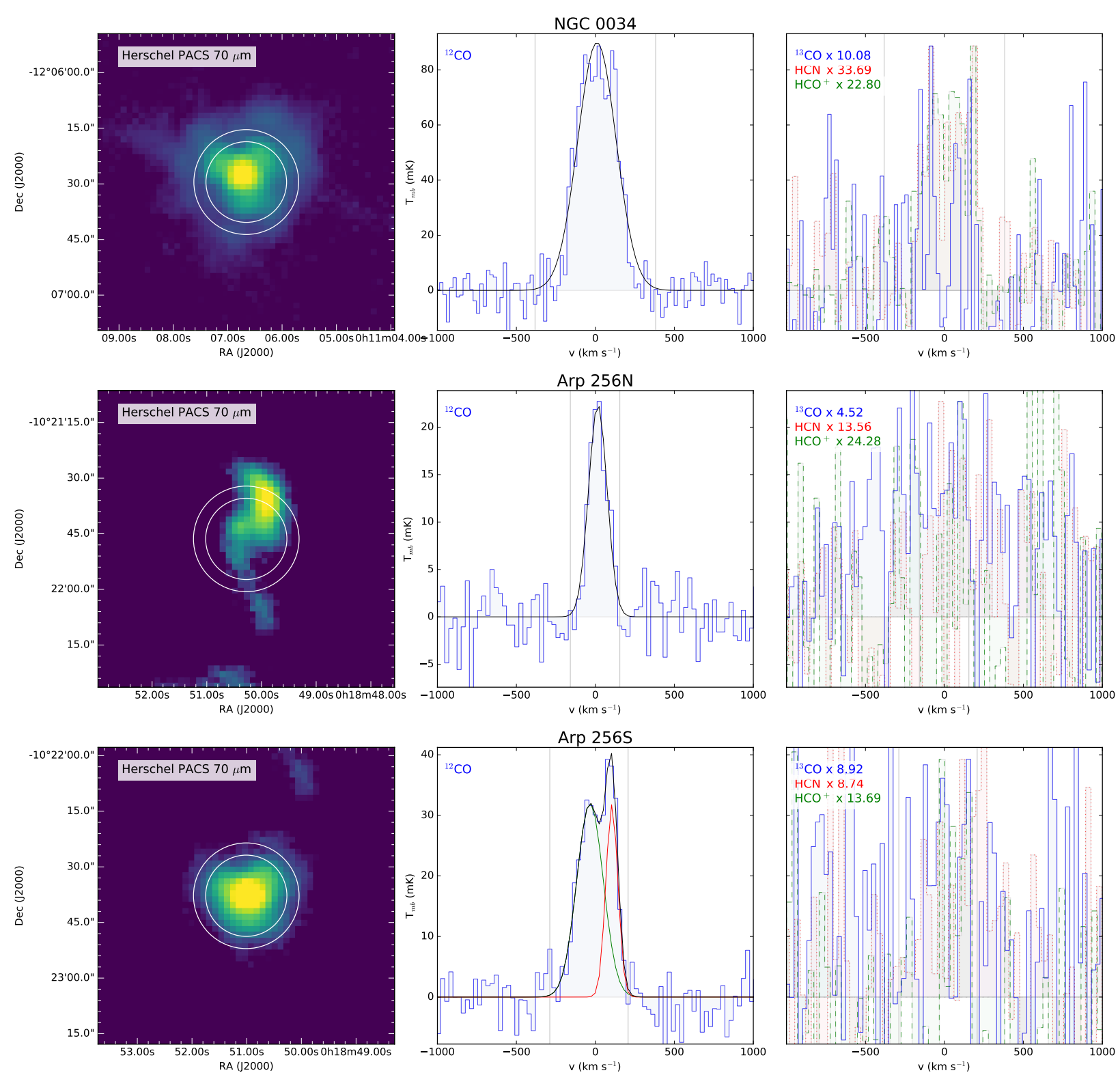

Fig. A.1. Herschel images and IRAM-30 m spectra. Figure 2 provides details. 

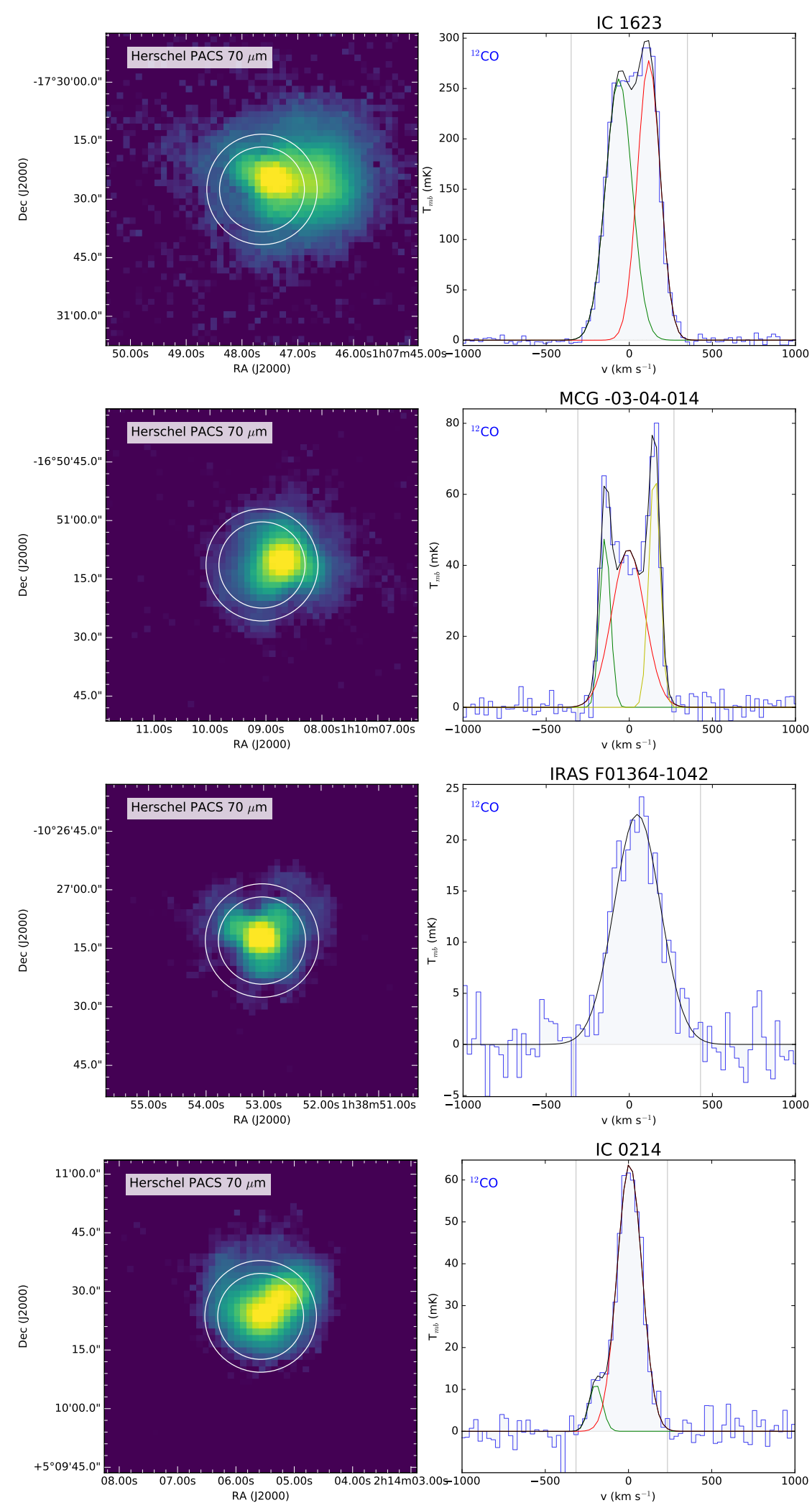
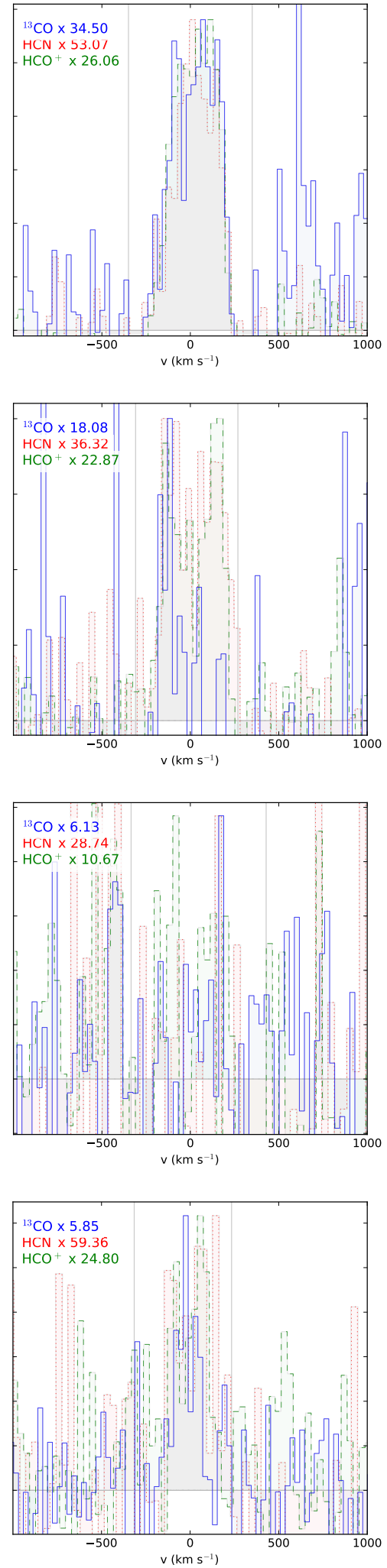

Fig. A.1. continued. 
R. Herrero-Illana et al.: Molecular gas and dust properties in (U)LIRGs from GOALS
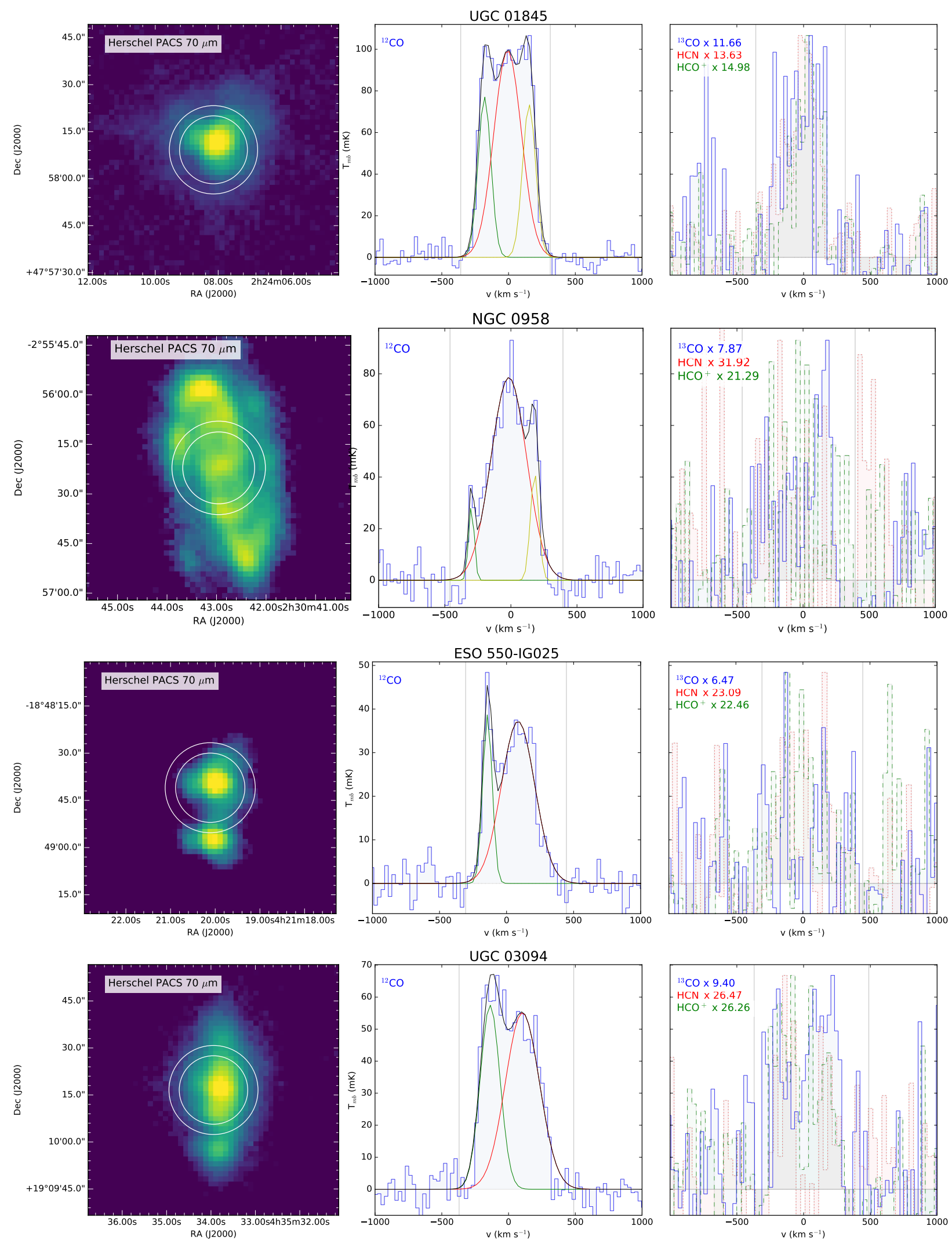

Fig. A.1. continued. 
A\&A 628, A71 (2019)
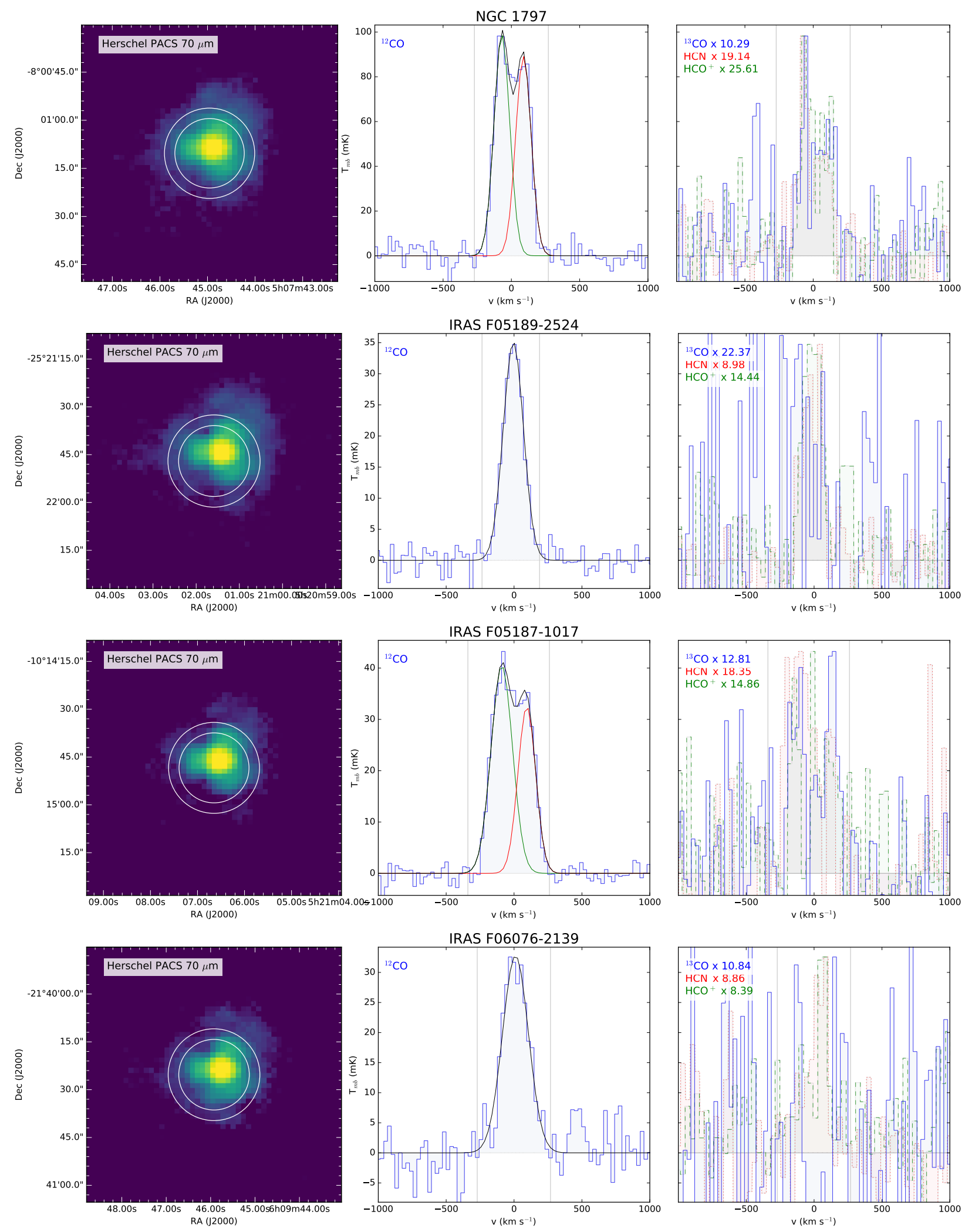

Fig. A.1. continued. 
R. Herrero-Illana et al.: Molecular gas and dust properties in (U)LIRGs from GOALS
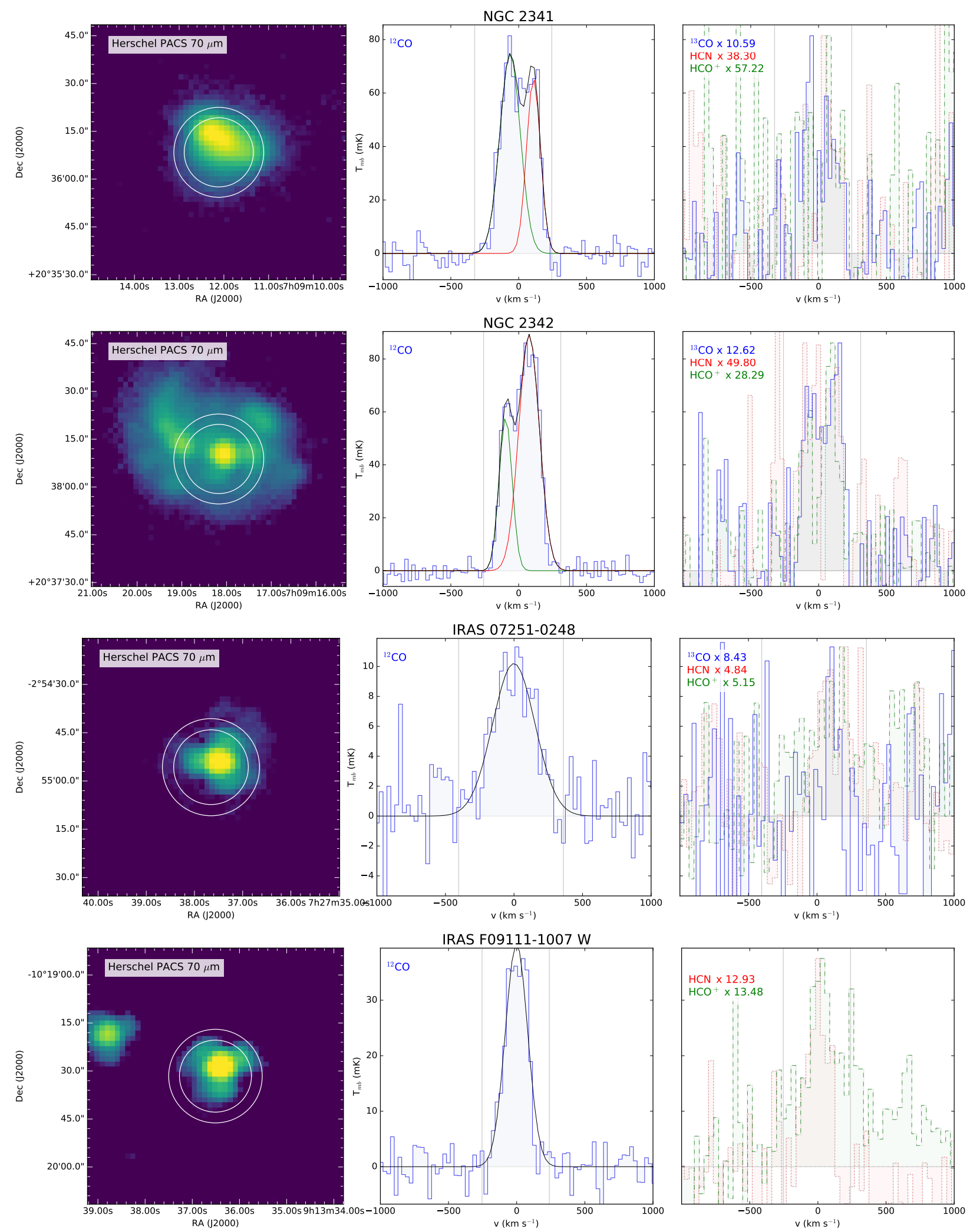

Fig. A.1. continued. 
A\&A 628, A71 (2019)
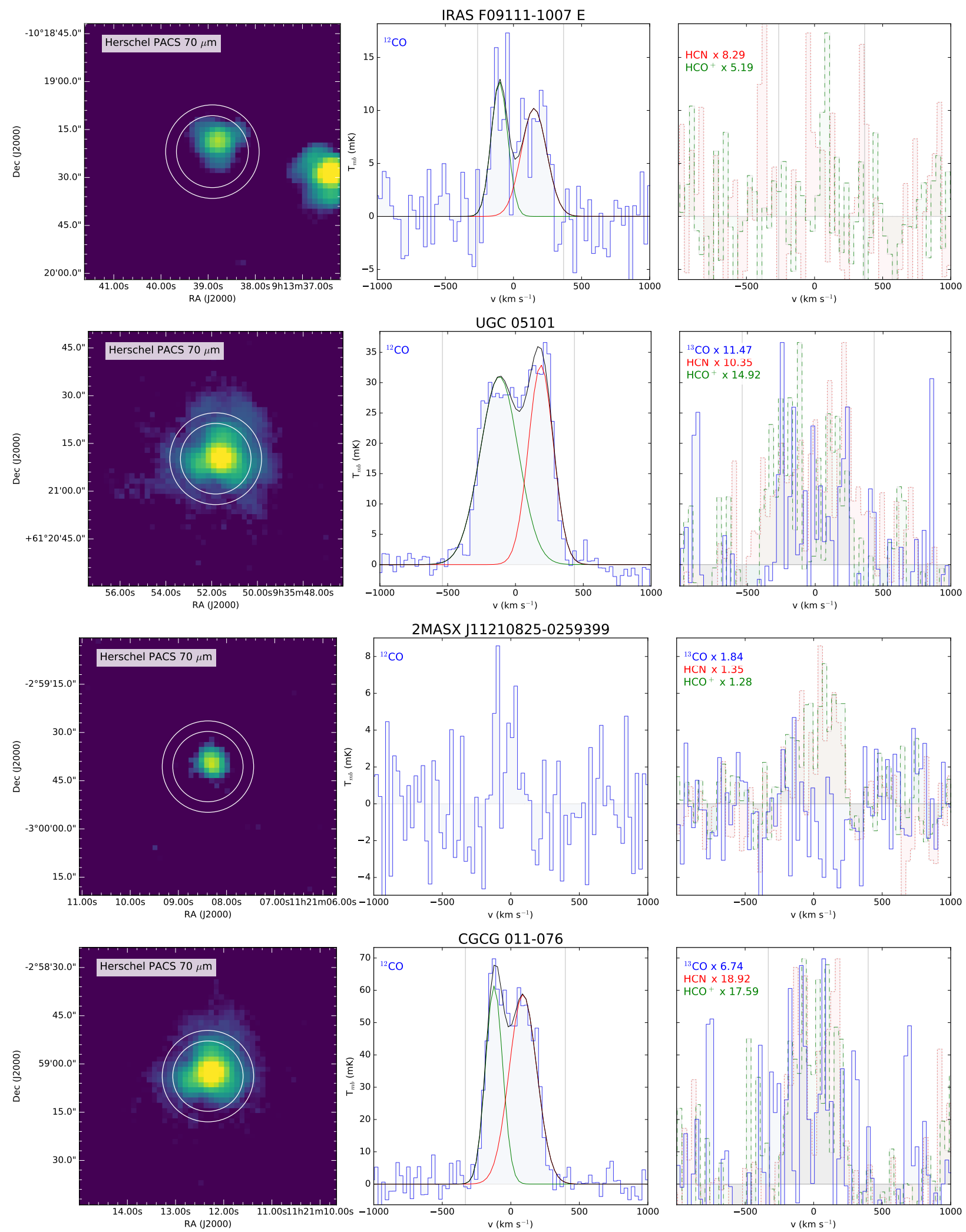

Fig. A.1. continued. 
R. Herrero-Illana et al.: Molecular gas and dust properties in (U)LIRGs from GOALS
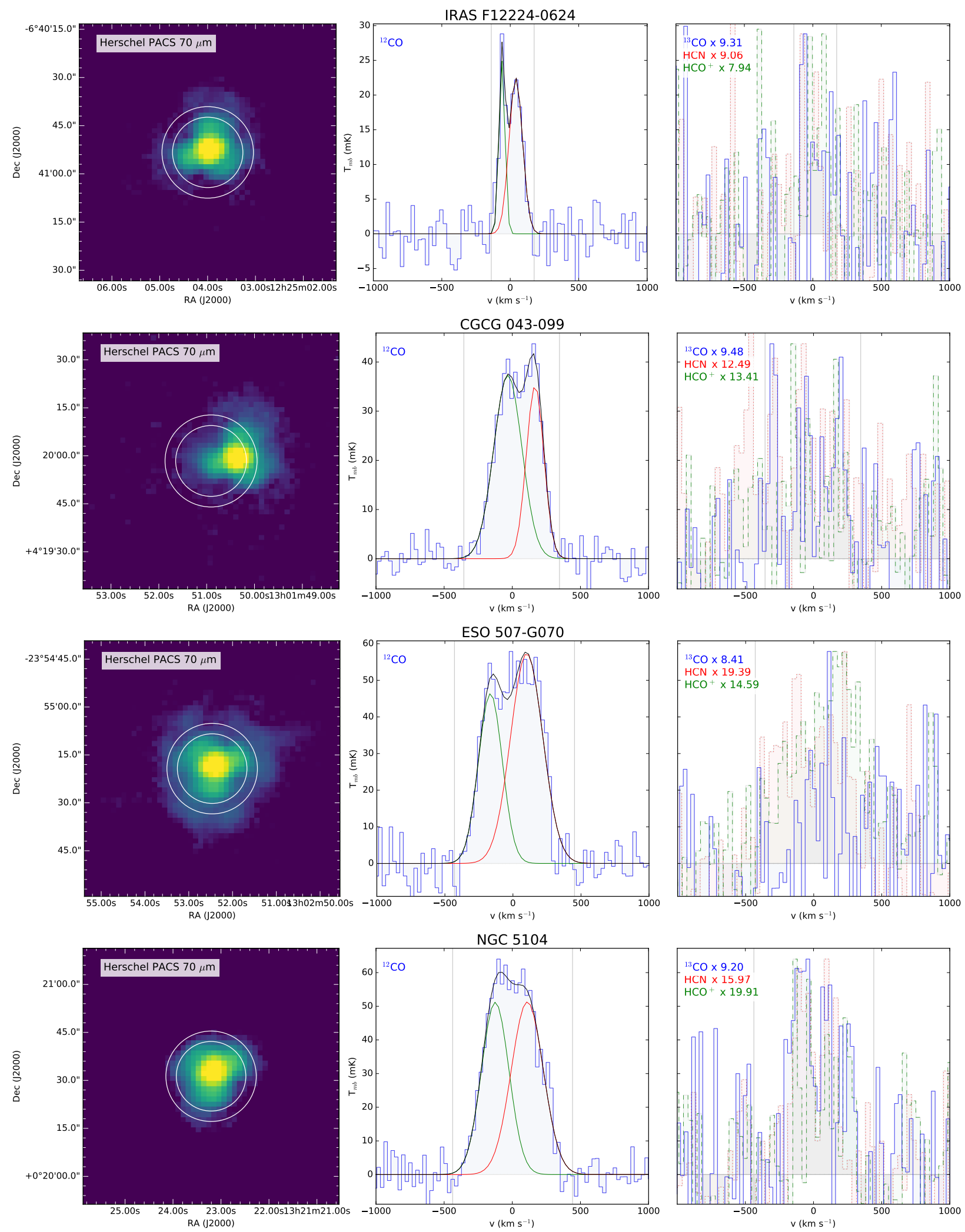

Fig. A.1. continued. 

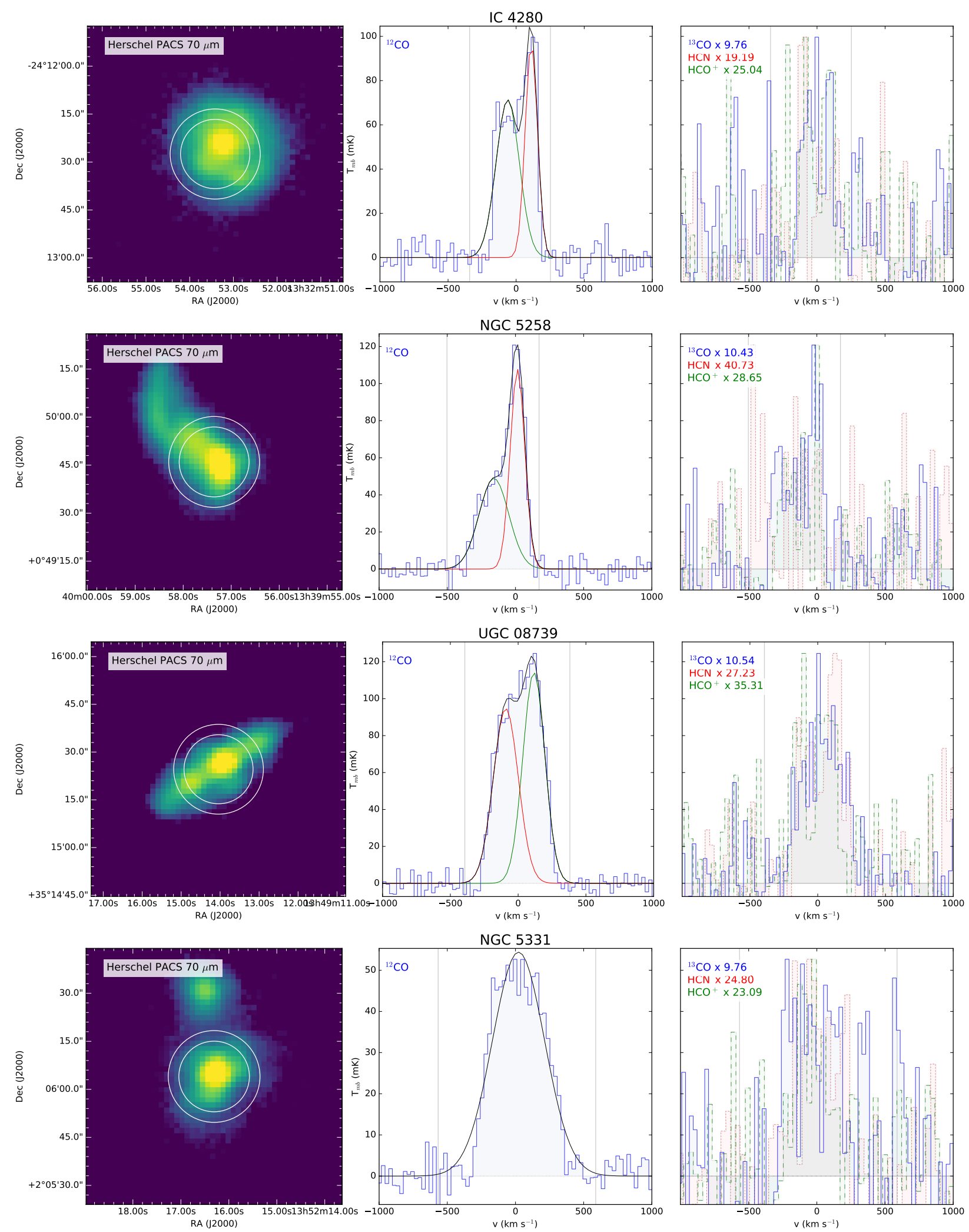

Fig. A.1. continued. 
R. Herrero-Illana et al.: Molecular gas and dust properties in (U)LIRGs from GOALS
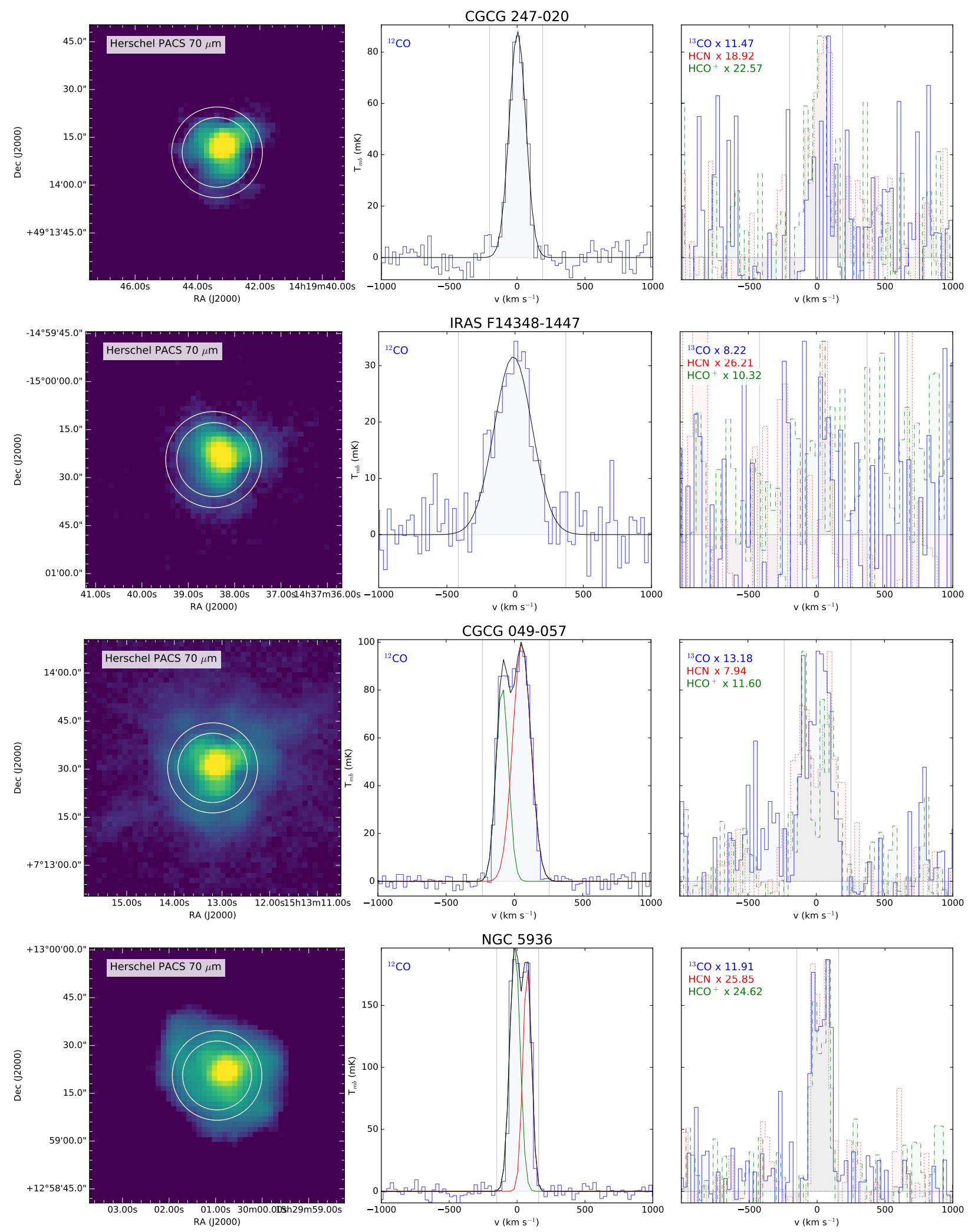

Fig. A.1. continued. 
A\&A 628, A71 (2019)
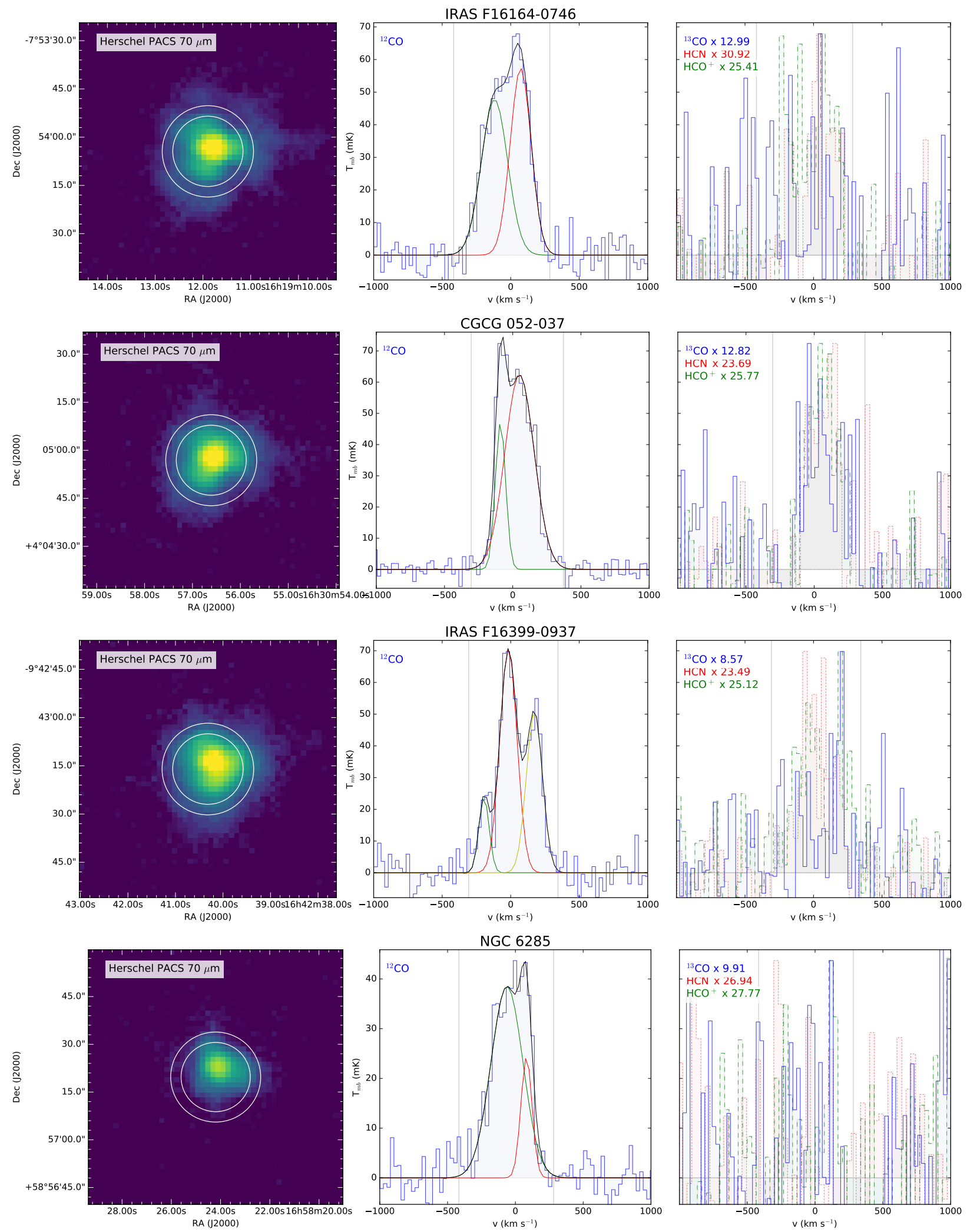

Fig. A.1. continued. 
R. Herrero-Illana et al.: Molecular gas and dust properties in (U)LIRGs from GOALS
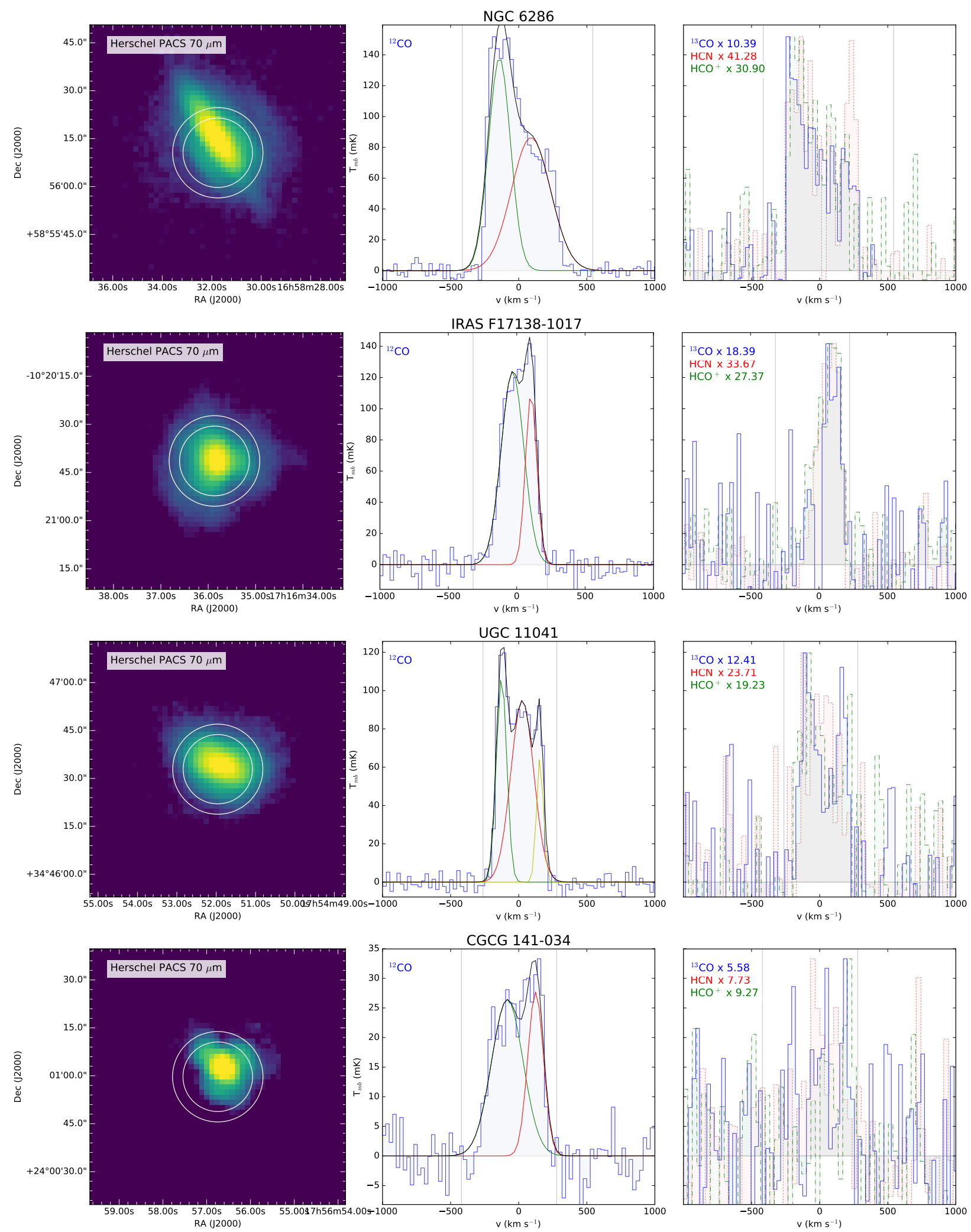

Fig. A.1. continued. 
A\&A 628, A71 (2019)
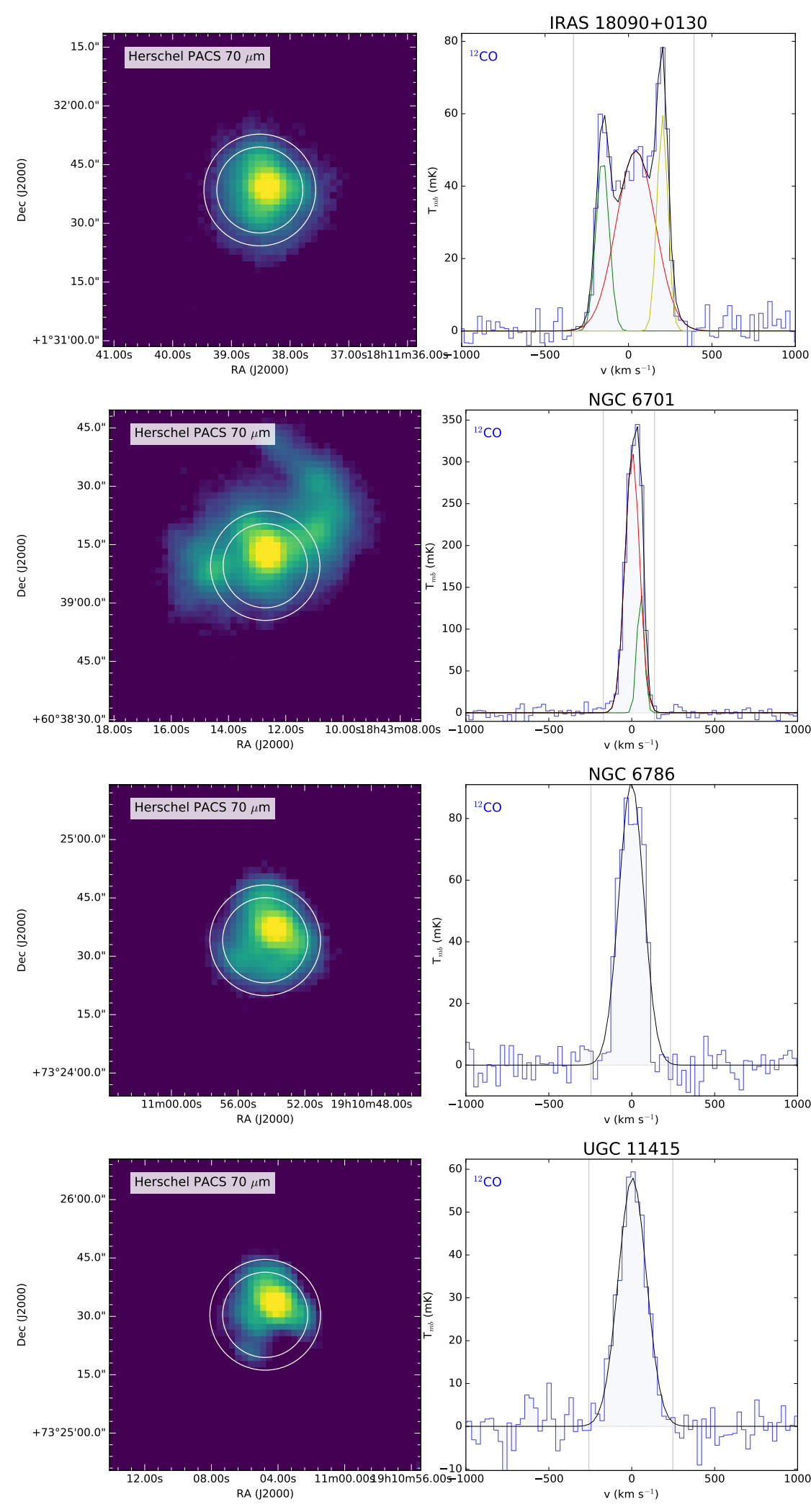

Fig. A.1. continued.
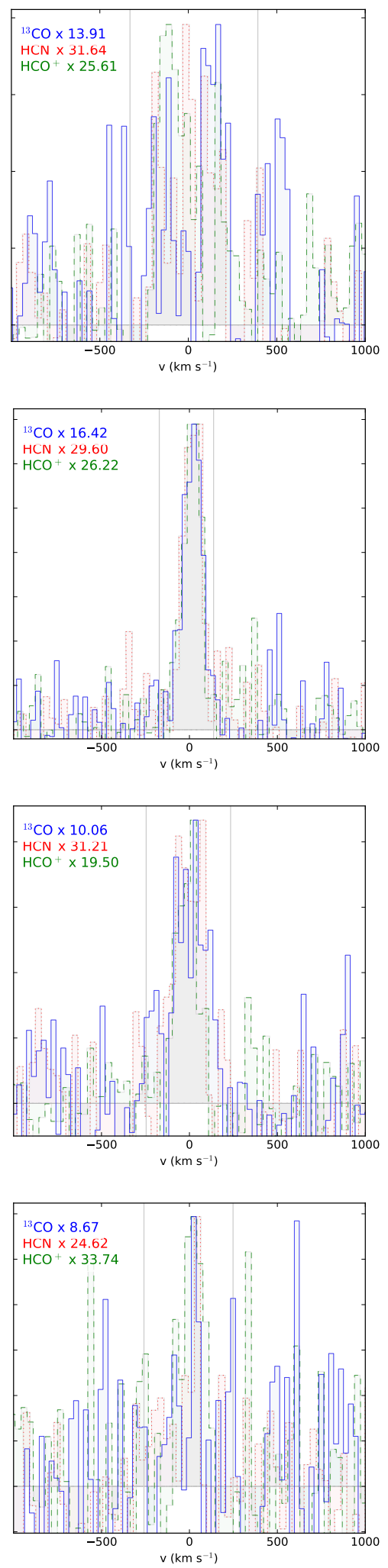
R. Herrero-Illana et al.: Molecular gas and dust properties in (U)LIRGs from GOALS
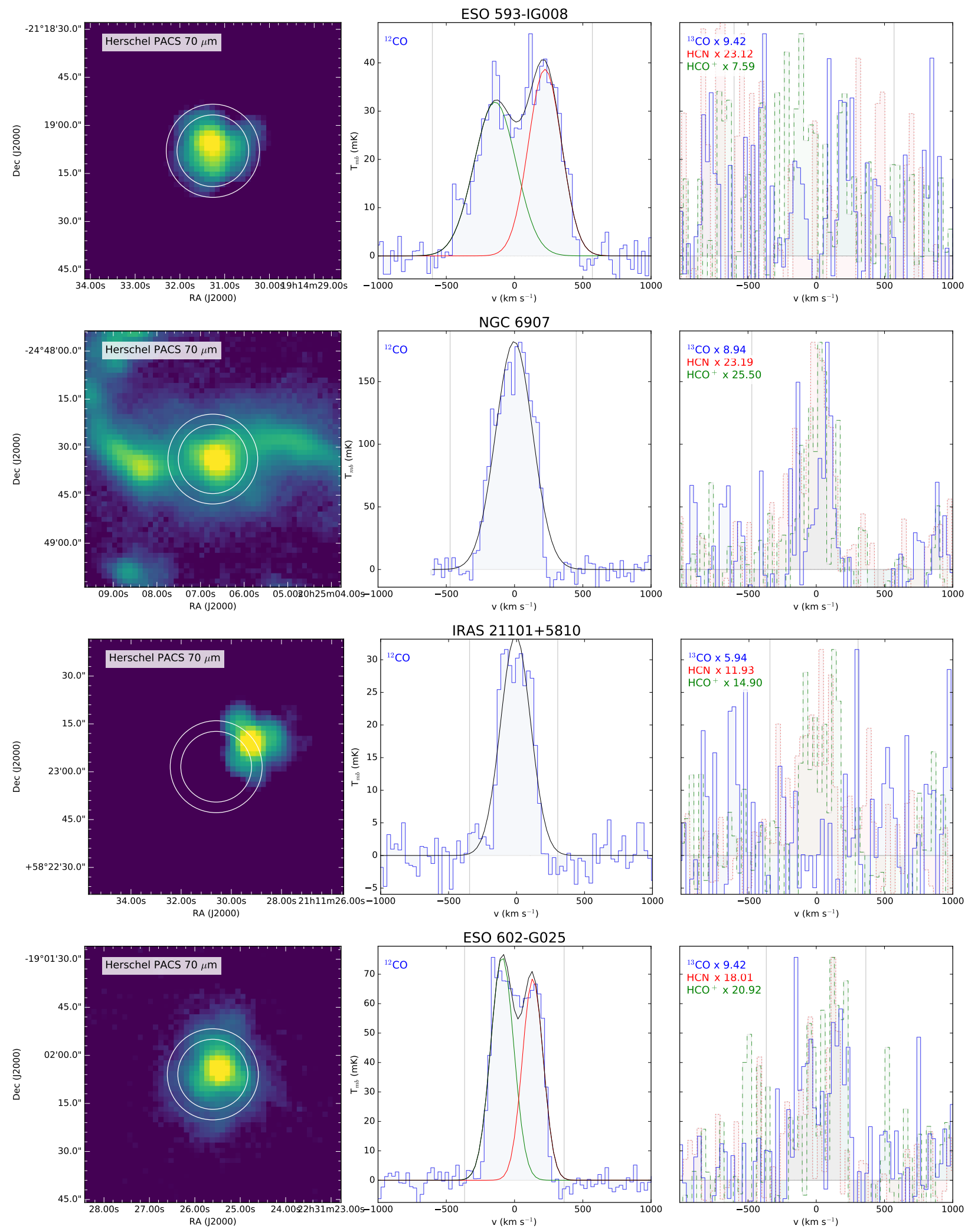

Fig. A.1. continued. 
A\&A 628, A71 (2019)
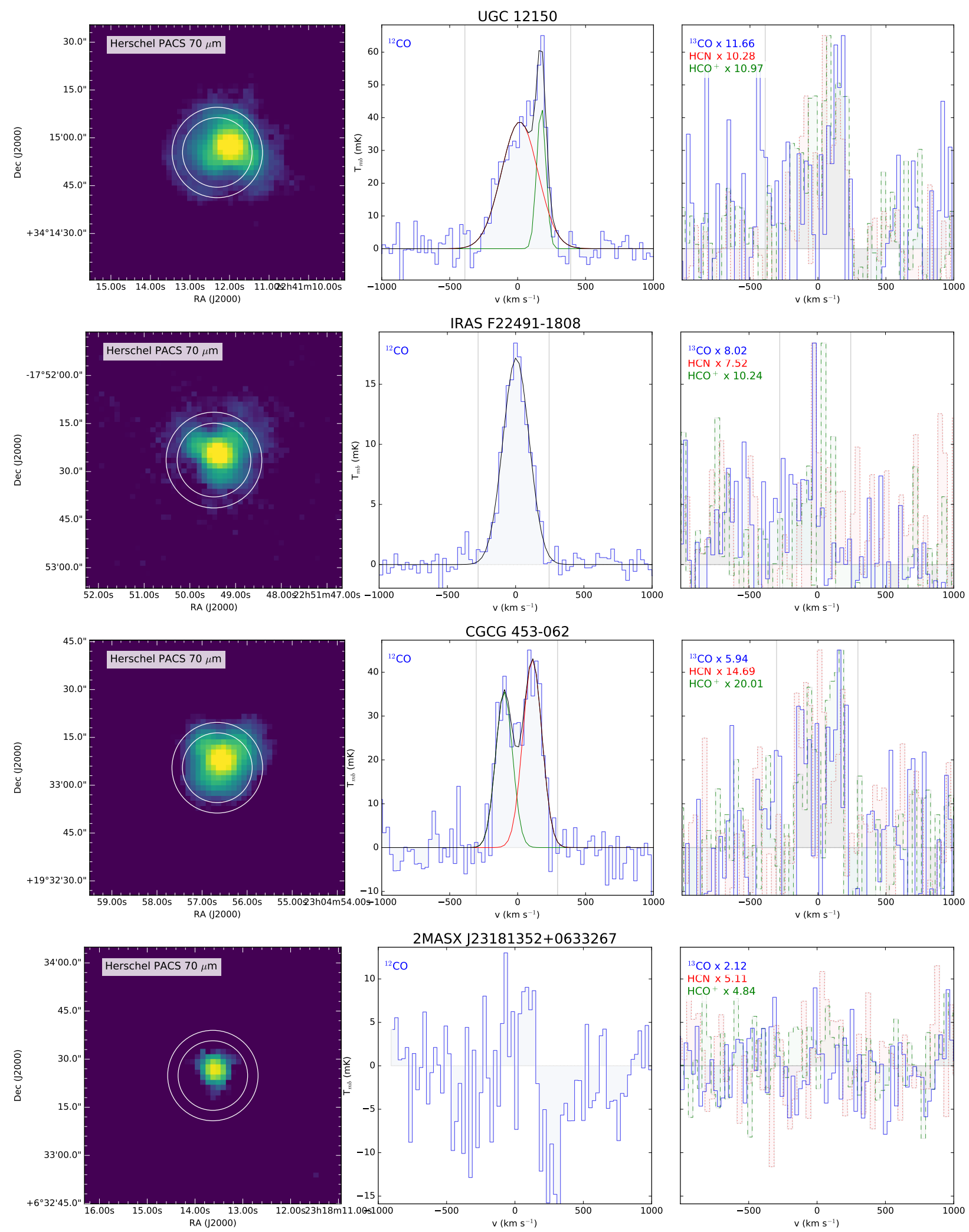

Fig. A.1. continued. 
Table A.1. Fitted Gaussian line components to the ${ }^{12} \mathrm{CO}$ spectra.

\begin{tabular}{|c|c|c|c|c|c|c|c|c|c|}
\hline \multirow[b]{2}{*}{ Source name } & \multicolumn{3}{|c|}{ First component } & \multicolumn{3}{|c|}{ Second component } & \multicolumn{3}{|c|}{ Third component } \\
\hline & $\begin{array}{l}\text { Position } \\
\left(\mathrm{km} \mathrm{s}^{-1}\right)\end{array}$ & $\begin{array}{c}F W H M \\
\left(\mathrm{~km} \mathrm{~s}^{-1}\right)\end{array}$ & $\begin{array}{c}\text { Peak } \\
\mathrm{mK}\end{array}$ & $\begin{array}{l}\text { Position } \\
\left(\mathrm{km} \mathrm{s}^{-1}\right)\end{array}$ & $\begin{array}{c}F W H M \\
\left(\mathrm{~km} \mathrm{~s}^{-1}\right)\end{array}$ & $\begin{array}{c}\text { Peak } \\
\mathrm{mK}\end{array}$ & $\begin{array}{l}\text { Position } \\
\left(\mathrm{km} \mathrm{s}^{-1}\right)\end{array}$ & $\begin{array}{c}F W H M \\
\left(\mathrm{~km} \mathrm{~s}^{-1}\right)\end{array}$ & $\begin{array}{c}\text { Peak } \\
\mathrm{mK} \\
\end{array}$ \\
\hline NGC 0034 & 10.3 & 285.9 & 89.9 & & $\ldots$ & $\ldots$ & $\ldots$ & $\ldots$ & $\ldots$ \\
\hline Arp $256 \mathrm{~N}$ & 15.7 & 137.2 & 22.4 & & & $\ldots$ & $\ldots$ & $\ldots$ & $\ldots$ \\
\hline Arp $256 \mathrm{~S}$ & -33.8 & 197.3 & 32.0 & 104.7 & 89.6 & 31.8 & $\ldots$ & $\ldots$ & $\ldots$ \\
\hline IC 1623 & -62.8 & 186.6 & 259.6 & 117.6 & 159.7 & 277.6 & $\ldots$ & $\ldots$ & $\ldots$ \\
\hline MCG -03-04-014 & -145.9 & 74.7 & 48.2 & -5.9 & 232.4 & 44.5 & 151.9 & 79.1 & 67.1 \\
\hline IRAS F01364-1042 & 48.1 & 326.8 & 22.5 & $\ldots$ & $\ldots$ & $\ldots$ & $\ldots$ & $\ldots$ & $\ldots$ \\
\hline IC 0214 & -197.0 & 93.8 & 11.3 & 6.3 & 176.1 & 63.9 & & $\ldots$ & . \\
\hline UGC 01845 & -179.9 & 112.8 & 77.1 & -6.7 & 242.7 & 99.4 & 154.2 & 115.3 & 73.5 \\
\hline NGC 0958 & -298.6 & 50.2 & 29.1 & -15.5 & 316.9 & 78.5 & 179.5 & 74.7 & 41.5 \\
\hline ESO 550-IG025 & -142.7 & 83.0 & 38.7 & 85.9 & 293.5 & 37.2 & $\ldots$ & $\ldots$ & $\ldots$ \\
\hline UGC 03094 & -138.0 & 174.3 & 57.5 & 103.9 & 300.8 & 55.1 & $\ldots$ & $\ldots$ & $\ldots$ \\
\hline NGC 1797 & -69.6 & 135.3 & 98.8 & 88.2 & 133.5 & 89.1 & $\ldots$ & $\ldots$ & $\ldots$ \\
\hline IRAS F05189-2524 & -5.1 & 175.8 & 35.1 & $\ldots$ & $\ldots$ & $\ldots$ & $\ldots$ & $\ldots$ & $\ldots$ \\
\hline IRAS F05187-1017 & -90.1 & 185.8 & 40.5 & 94.0 & 153.3 & 32.5 & $\ldots$ & $\ldots$ & $\ldots$ \\
\hline IRAS F06076-2139 & 10.5 & 225.3 & 32.8 & & & & $\ldots$ & $\ldots$ & $\ldots$ \\
\hline NGC 2341 & -63.3 & 177.4 & 74.7 & 109.2 & 120.6 & 65.3 & $\ldots$ & $\ldots$ & $\ldots$ \\
\hline NGC 2342 & -96.3 & 111.7 & 58.0 & 76.6 & 178.3 & 89.3 & $\ldots$ & $\ldots$ & $\ldots$ \\
\hline IRAS 07251-0248 & -0.9 & 363.1 & 10.2 & $\ldots$ & $\ldots$ & $\ldots$ & $\ldots$ & $\ldots$ & $\ldots$ \\
\hline IRAS F09111-1007 W & -1.2 & 193.2 & 40.2 & $\ldots$ & $\ldots$ & $\ldots$ & $\ldots$ & $\ldots$ & $\ldots$ \\
\hline IRAS F09111-1007 E & -102.9 & 141.5 & 12.7 & 150.6 & 218.2 & 10.2 & $\ldots$ & $\ldots$ & $\ldots$ \\
\hline UGC 05101 & -118.9 & 330.9 & 31.0 & 187.4 & 216.1 & 32.9 & $\ldots$ & $\ldots$ & $\ldots$ \\
\hline 2MASX J11210825-0259399 & $\ldots$ & & $\ldots$ & $\ldots$ & & & $\ldots$ & $\ldots$ & $\ldots$ \\
\hline CGCG 011-076 & -121.9 & 146.7 & 61.4 & 89.0 & 236.8 & 58.7 & $\ldots$ & $\ldots$ & $\ldots$ \\
\hline IRAS F12224-0624 & -60.2 & 51.8 & 24.9 & 40.5 & 115.7 & 22.5 & $\ldots$ & $\ldots$ & $\ldots$ \\
\hline CGCG 043-099 & -32.5 & 243.3 & 37.3 & 167.1 & 151.0 & 35.2 & $\ldots$ & $\ldots$ & $\ldots$ \\
\hline ESO 507-G070 & -165.5 & 205.9 & 46.4 & 101.1 & 278.7 & 57.3 & $\ldots$ & $\ldots$ & $\ldots$ \\
\hline NGC 5104 & -125.2 & 237.7 & 51.2 & 110.0 & 276.8 & 51.3 & $\ldots$ & $\ldots$ & $\ldots$ \\
\hline IC 4280 & -61.7 & 200.9 & 71.3 & 111.7 & 105.7 & 96.5 & $\ldots$ & $\ldots$ & $\ldots$ \\
\hline NGC 5258 & -158.1 & 256.1 & 48.9 & 15.0 & 125.5 & 107.7 & $\ldots$ & $\ldots$ & $\ldots$ \\
\hline UGC 08739 & 116.3 & 190.6 & 114.3 & -90.7 & 216.9 & 94.7 & $\ldots$ & $\ldots$ & $\ldots$ \\
\hline NGC 5331 & 22.3 & 449.5 & 54.4 & $\ldots$ & $\ldots$ & $\ldots$ & $\ldots$ & $\ldots$ & $\ldots$ \\
\hline CGCG 247-020 & 3.4 & 143.8 & 88.1 & $\ldots$ & $\ldots$ & $\ldots$ & $\ldots$ & $\ldots$ & $\ldots$ \\
\hline IRAS F14348-1447 & -13.5 & 326.9 & 31.6 & $\ldots$ & $\ldots$ & $\ldots$ & $\ldots$ & $\ldots$ & $\ldots$ \\
\hline CGCG 049-057 & -89.1 & 106.2 & 81.6 & 53.8 & 155.4 & 99.6 & $\ldots$ & $\ldots$ & $\ldots$ \\
\hline NGC 5936 & -16.1 & 88.6 & 200.3 & 75.0 & 74.8 & 182.3 & $\ldots$ & $\ldots$ & $\ldots$ \\
\hline IRAS F16164-0746 & -118.1 & 225.0 & 47.8 & 72.7 & 178.5 & 57.3 & $\ldots$ & $\ldots$ & $\ldots$ \\
\hline CGCG 052-037 & -89.9 & 88.9 & 47.1 & 50.0 & 252.4 & 62.4 & $\ldots$ & $\ldots$ & $\ldots$ \\
\hline IRAS F16399-0937 & -194.7 & 88.6 & 23.7 & -16.7 & 147.5 & 70.6 & 173.2 & 138.6 & 50.6 \\
\hline NGC 6285 & -62.0 & 277.1 & 38.5 & 81.8 & 97.0 & 24.3 & $\ldots$ & $\ldots$ & $\ldots$ \\
\hline NGC 6286 & -141.0 & 189.1 & 138.2 & 89.9 & 338.7 & 86.3 & $\ldots$ & $\ldots$ & $\ldots$ \\
\hline IRAS F17138-1017 & -30.6 & 195.1 & 123.7 & 105.3 & 97.1 & 109.9 & $\ldots$ & $\ldots$ & \\
\hline UGC 11041 & -125.7 & 88.9 & 107.5 & 26.3 & 198.4 & 94.9 & 155.8 & 59.2 & 65.1 \\
\hline CGCG 141-034 & -83.6 & 272.6 & 26.4 & 124.0 & 135.4 & 27.7 & $\ldots$ & $\ldots$ & $\ldots$ \\
\hline IRAS 18090+0130 & -155.4 & 91.6 & 48.3 & 43.8 & 274.0 & 49.8 & 203.3 & 75.8 & 59.6 \\
\hline NGC 6701 & 53.2 & 50.1 & 147.9 & 3.3 & 102.8 & 311.5 & $\ldots$ & $\ldots$ & $\ldots$ \\
\hline NGC 6786 & -2.3 & 174.8 & 91.9 & $\ldots$ & $\ldots$ & $\ldots$ & $\ldots$ & $\ldots$ & $\ldots$ \\
\hline UGC 11415 & 4.0 & 199.5 & 58.0 & $\ldots$ & $\ldots$ & $\ldots$ & $\ldots$ & $\ldots$ & $\ldots$ \\
\hline ESO 593-IG008 & -142.2 & 361.1 & 31.9 & 222.7 & 284.8 & 38.7 & $\ldots$ & $\ldots$ & $\ldots$ \\
\hline NGC 6907 & -2.8 & 317.3 & 182.1 & $\ldots$ & $\ldots$ & $\ldots$ & $\ldots$ & $\ldots$ & $\ldots$ \\
\hline IRAS $21101+5810$ & -6.7 & 259.0 & 33.8 & $\ldots$ & $\ldots$ & $\ldots$ & $\ldots$ & $\ldots$ & $\ldots$ \\
\hline ESO 602-G025 & -90.3 & 198.5 & 75.9 & 133.6 & 180.1 & 68.5 & $\ldots$ & $\ldots$ & $\ldots$ \\
\hline UGC 12150 & 174.2 & 78.6 & 43.7 & 15.6 & 316.4 & 38.7 & $\ldots$ & $\ldots$ & $\ldots$ \\
\hline IRAS F22491-1808 & 3.5 & 226.4 & 17.2 & $\ldots$ & $\ldots$ & $\ldots$ & $\ldots$ & $\ldots$ & $\ldots$ \\
\hline CGCG 453-062 & -99.3 & 145.5 & 35.5 & 108.9 & 164.3 & 43.0 & $\ldots$ & $\ldots$ & $\ldots$ \\
\hline 2MASX J23181352+0633267 & $\ldots$ & $\ldots$ & $\ldots$ & $\ldots$ & $\ldots$ & $\ldots$ & $\ldots$ & $\ldots$ & $\ldots$ \\
\hline
\end{tabular}




\section{Appendix B: Modified blackbody model SED fitting}

Assuming that all dust grains have the same size and and composition, their emission can be modeled by an optically thin modified blackbody,

$S(v)=\frac{Q(v) B(v, T) N \sigma}{D^{2}}$,

where $S(v)$ is the flux density, $Q(v)$ is the emissivity, $B(v, T)$ is the blackbody intensity, $N$ and $\sigma$ are the number of identical dust particles and their cross section, respectively, and $D$ is the distance to the dust cloud.

We have used Herschel data from both PACS $(70,100$, and $170 \mu \mathrm{m})$ and SPIRE (250 and $350 \mu \mathrm{m}$, excluding $500 \mu \mathrm{m}$ for having a too coarse resolution) to construct the SED of each source. To do so, we smoothed the images to the FWHM of the IRAM-30 m observations, except for the $350 \mu \mathrm{m}$ images, which already have an angular resolution comparable with the IRAM$30 \mathrm{~m}$ beam. We then used a $\chi^{2}$ minimization method to obtain the best model. The emissivity index $\beta$ (where $\kappa(v) \propto \nu^{\beta}$ ) was fixed to a value of $\beta=1.8$, to avoid degeneracies between $\beta$ and $T_{\text {dust }}$ in $\chi^{2}$ minimization fits (Blain et al. 2003).

We can rewrite Eq. (B.1) in terms of the dust mass, $M_{\mathrm{d}}$, as

$M_{\mathrm{d}}=\frac{S\left(v_{\mathrm{obs}}\right) D^{2}}{\kappa\left(v_{\text {rest }}\right) B\left(v_{\text {rest }}, T\right)(1+z)}$,

where $\kappa(v)$ is the so-called grain absorption cross section per unit mass or mass absorption coefficient. This parameter is highly uncertain (see, e.g., Draine \& Lee 1984; Davies et al. 2012; Bianchi 2013). For our model we have adopted an intermediate value of $\kappa(250 \mu \mathrm{m})=0.48 \mathrm{~m}^{2} \mathrm{~kg}^{-1}$. Complementary to the dust masses and temperatures, we have derived $L_{\mathrm{FIR}}$ from direct integration below the fitted curve between 42.5 and $122.5 \mu \mathrm{m}$. Figure B.1 shows an example for the fit in the galaxy NGC 0958.

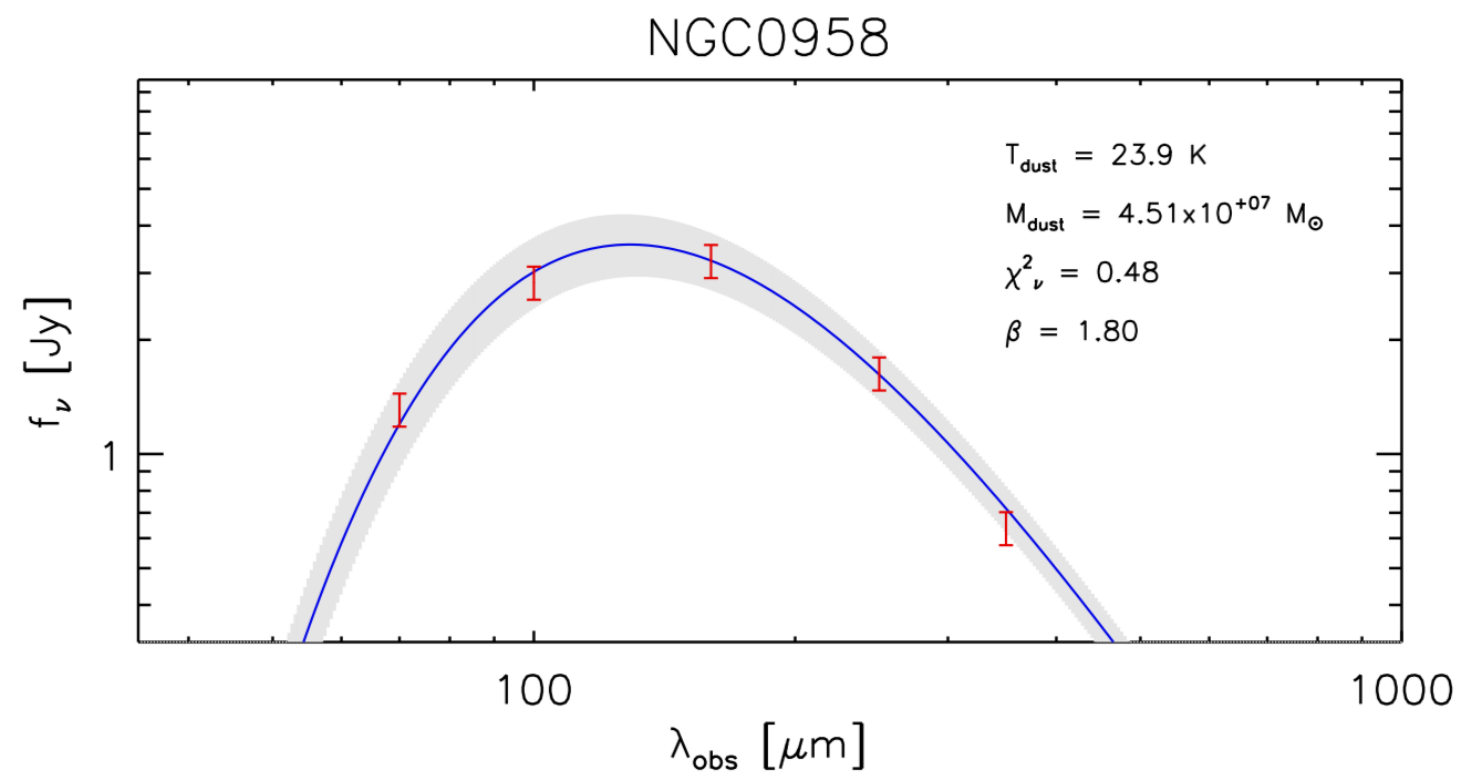

Fig. B.1. SED fitting for NGC 0958. Red lines show the Herschel photometry with its uncertainty. The best model is shown as a solid blue line and the shaded area represents the uncertainty in the fit. 